\title{
Quantitative Analysis of Software Approximate Correctness
}

\author{
Yanfang Ma \\ School of Computer Science and Technology, Huaibei Normal University, Huaibei 235000, China \\ Correspondence should be addressed to Yanfang Ma; myf1978@gmail.com
}

Received 6 November 2014; Accepted 14 June 2015

Academic Editor: Fabio Tramontana

Copyright (C) 2015 Yanfang Ma. This is an open access article distributed under the Creative Commons Attribution License, which permits unrestricted use, distribution, and reproduction in any medium, provided the original work is properly cited.

Parameterized bisimulation provides an abstract description of software correctness. In real world situations, however, many software products are approximately correct. To characterize the approximate correctness, we generalize the parameterized bisimulation to numerical version and probabilistic setting. First, we propose the definition of the parameterized bisimulation index that expresses the degree to which a binary relation is parameterized bisimulation. Then, $\lambda$-parameterized bisimulation over environment $e$ and its substitutivity laws are presented. Finally, $\lambda$-parameterized probabilistic bisimulation is established to describe complicated software products with probabilistic phenomena.

\section{Introduction}

Correctness is a key feature of software trustworthiness [1-3], which can be abstracted by using various behavior equivalences between processes, such as (strong and weak) bisimilarity, trace equivalence, testing equivalence, and failure equivalence [4-6]. Specification and implementation of software are considered as two processes. If a certain behavior equivalence exists between specification and implementation, then the software is considered as correctness. Thus a certain behavior equivalence must be established between specification and implementation to prove software correctness.

However, the prerequisites for successful application of software products may not always hold when they are actually running on the computers. As physical devices, computers cannot be assumed to behave reliably. In addition, standard implementations at best approximate the formal definition of semantics. Ying [7] proposed strong/weak bisimulation indexes to establish the approximate description between specification and implementation. The proposed indexes characterize the degree to which a binary relation between processes is strong/weak bisimulations. Ying and Wirsing [8] presented the strong/weak bisimulation limits and obtained the strong/weak bisimulation topologies to describe that the sequence of implementations can be treated as an evolution toward the specification. Girard and Pappas [9] defined a hierarchy of approximate pseudometric between two systems that quantifies the qualities of the approximations. To verify whether a program behaved as desired, Henzinger [10] introduced quantitative fitness measures for programs, particularly to measure the function, performance, and robustness of reactive programs such as concurrent processes. To compare these existing quantitative models of program approximate correctness, Fahrenberg and Legay [11] presented a distanceagnostic approach to quantify the verification. They defined a spectrum of different interesting system distances that corresponds to the given trace distance.

In fact, some complicated software products contain probabilistic phenomena. These software products can be abstracted as probabilistic processes. Similarly, many quantitative models based on probabilistic processes [12] have existed to obtain the degree to which implementations satisfy their specification. For example, Giacalone et al. $[13,14]$ presented $\varepsilon$-bisimulation equivalence relation over deterministic probabilistic processes and proposed a kind of measure model to describe the degree of similarity among probabilistic processes. This measure is defined based on the probability differences of the processes that execute the same action. Song et al. [15] proposed a measure model according to the probability of the processes that performs the same trace with a discount factor. Deng et al. [16] defined 
state-metrics as a natural extension of bisimulation from nonquantitative systems to quantitative ones over actionlabeled quantitative systems. Alves de Medeiros et al. [17] built a measure relation based on the observable actions of processes. Abate [18] also established an approximate metric based on probabilistic bisimulation.

However, the running of a software depends on its environment. The environment should be considered when the approximation degree between specification and implementation is discussed. The influences of the environment are absent in the existing quantitative models. In [19], Larsen and Skou presented two-thirds bisimulation based on probabilistic transition systems to characterize that two processes are undistinguished when they have the same sets of observations for all tests. If an environment is considered as a set of actions [20], then two-thirds bisimulation expresses the relation in which the process refuses the environment. We proposed two-thirds simulation index and established a measure model to describe the degree of approximation among processes [21]. In $\pi$-calculus [22] and applied $\pi$-calculus [23], the observation equivalences were researched. And the influence of environment on the execution of software was considered as well. In [22], a process context is speaking a process expression containing a hole. In [23], the contexts may be used to represent the adversarial environment in which a process is run. The environment provides the data that the process inputs and consumes the data that it outputs.

Larsen [24] presented parameterized bisimulation equivalence to obtain flexible hierarchic development methods. In the work of Larsen, bisimulation equivalence is parameterized with information about context called environment. Environment $e$ is considered as an object that consumes the actions produced by a process in that environment. However, the abilities of environment to consume actions might be limited. Suppose that $P$ is a process, and it can execute action $\alpha$ to next process $P^{\prime}$; that is, $P \stackrel{\alpha}{\rightarrow} P^{\prime}$. However, $e$ cannot consume the action $\alpha$; then derivation $P \stackrel{\alpha}{\rightarrow} P^{\prime}$ will never be considered when $P$ is executed in environment $e$. If $P$ and $Q$ both perform the same action for all transitions of $e$, then we can determine $P \sim_{e} Q$. In particular, strong bisimulation in CCS (Communication and Concurrency Systems) model is generalized by parameterized bisimulation equivalence. Parameterized limit bisimulation and parameterized bisimulation limit were proposed in $[25,26]$ to describe the infinite evolution mechanism.

The conditions possessing the same observable actions consumed by the environment are rigorous when we choose parameterized bisimulation to verify software correctness. Sometimes we can determine that two processes fail to meet these conditions. However, these processes are still close to parameterized bisimulation in the sense that whenever a process can execute an action of environment consumption, another process can produce an action that is different from but highly similar to the observable action that the first process executed. Alternatively, another process can perform an action that is highly similar to the observable action that the first process made whenever a process can produce an action that is different from the action of the environment consuming.

The aim of this study is to build mathematical tools that are suitable for describing this kind of approximate parameterized bisimulation. First, we propose parameterized bisimulation index over environment $e$ in order to describe the degree to which binary relation $R_{e}$ is a parameterized bisimulation. Then we define $\lambda$-parameterized bisimulation and discuss algebraic properties. We specially prove the congruence of $\lambda$-parameterized bisimulation under various operators. Finally, in order to describe the characterization of software with probabilistic information, we also extend parameterized bisimulation to probabilistic setting and propose the approximate parameterized probabilistic bisimulation.

Compared with the main focuses of $[7,8,21]$, the main focus of our work is on parameterized bisimulation. In $[7,8]$, the set of labels in a labeled transition system is equipped with a metric. Given a binary relation between processes, the degree to which the relation is bisimulation is defined. Similar to $[7,21]$, we also equip the set of actions with a metric. Parameterized bisimulation that includes the information about context is different from bisimulation. For every environment $e, R_{e}$ is a binary relation between processes. Therefore, in order to obtain the approximate parameterized bisimulation, we need to establish the bisimulation index for every environment $e$. We consider two cases to obtain the bisimulation index for an environment. One case is that when the environment consumes an action, a process can accept this action and another process cannot accept this action. Another case is that when the environment has the transition with an action, two processes cannot both accept this action. Therefore, our definition about bisimulation index on the environment is different from the definition of bisimulation index in [7]. Furthermore, we establish the $\lambda$-parameterized bisimulation on the environment $e$. In order to obtain the hierarchic development and modular decomposition of software, similar to $[7,21]$, we also consider the substitutivity laws of $\lambda$-parameterized bisimulation on the environment under various combinators.

Meanwhile, we notice that many metric models are proposed based on the difference of probabilities in which two processes execute the same action [16]. But the influence of environment was not considered in these models. In order to describe the approximation of the complicated software with probabilistic information, we extend parameterized bisimulation to probabilistic setting in order to reflect the environment. First, we extend the environment transition system to probabilistic case. Then, we define parameterized probabilistic bisimulation. Finally, we obtain the $\lambda$ parameterized probabilistic bisimulation based on the probabilities that the environment consumes an action and the processes perform the same action. This point is similar to [14, 16]. Our method is different from the method in [18]. In [18], the state space is equipped with a rich structure, whereas the metric is characterized by probabilistic conditional kernels.

In Section 2, we recall the syntax of CCS and parameterized bisimulation. Parameterized bisimulation index over environment $e$ and $\lambda$-parameterized bisimulation are 
defined in Section 3. Their some algebraic properties are researched in Section 3. In Section 4, the substitutivity laws of $\lambda$-parameterized bisimulation under various operators are proved. In Section 5, parameterized probabilistic bisimulation is proposed and $\lambda$-parameterized probabilistic bisimulation is defined. Furthermore, the congruence of $\lambda$-parameterized probabilistic bisimulation is proved. Our conclusions and future work are presented in Section 6.

\section{Preliminaries}

2.1. CCS Summary. This section recalls some fundamental concepts and the results of process calculus needed in the subsequent sections. The following definitions mainly come from the book by Ying [27].

We introduce the names $\mathscr{A}$, the conames $\overline{\mathscr{A}}$, and labels $\Gamma=\mathscr{A} \cup \overline{\mathscr{A}} \cdot a, b, \ldots$ range over $\mathscr{A}, \bar{a}, \bar{b}, \ldots$ range over $\overline{\mathscr{A}}, l, l^{\prime}, \ldots$ range over $\Gamma$ is defined. We also introduce the silent or perfect action $\tau$. Act $=\Gamma \cup\{\tau\}$ is defined as the set of actions, whereas $\alpha, \beta$ are defined range over Act. Furthermore, we introduce set $\aleph$ of process variables and set $\mathscr{K}$ of processes constants. Mapping $f: \Gamma \rightarrow \Gamma$ is a relabeling function if $f(\bar{l})=\overline{f(l)}$ for every $l \in \Gamma$. We may extend relabeling function $f$ to be a mapping from Act to itself by decreeing that $f(\tau)=\tau$. The syntax of the basic process calculus is presented in the following definition.

Definition 1 (process expression [28]). The class $\partial$ of process expressions is the smallest class of symbol strings that satisfies the following conditions:

(1) $\aleph, \mathscr{K} \in \partial$.

(2) If $\alpha \in$ Act and $E \in \partial$, then $\alpha . E \in \partial$.

(3) If $I$ is an indexing set and $E_{i} \in \partial(i \in I)$, then $\sum_{i \in I} E_{i} \in$ $\partial$.

(4) If $E_{1}, E_{2} \in \partial$, then $E_{1} \mid E_{2} \in \partial$.

(5) If $E \in \partial$ and $L \subseteq \Gamma$, then $E \backslash L \in \partial$.

(6) If $E \in \partial$ and $f$ is a relabeling function, then $E[f] \in \partial$.

The process expressions without process variables are called processes and the class of processes is denoted by $\mathscr{P}$. For any $A \in \Re$, we assume that there is a defining equation $A \stackrel{\text { def }}{=} P_{A}$, such as $A=P_{A}=l \cdot A \in \mathscr{P}$. Constants provide us a mechanism of recursion in the process calculus.

The transitional semantics of the basic calculus is presented in the style of Plotkin's structural operational semantics [29]. We have the following definition.

Definition 2 (labeled transition system [7]). Let ( $\partial$, Act, $\{\stackrel{\alpha}{\rightarrow}$ : $\alpha \in \mathrm{Act}\}$ ) be a labeled transition system, where the transition relations $\stackrel{\alpha}{\rightarrow}(\alpha \in \mathrm{Act})$ are presented by the following rules:

$$
\begin{gathered}
\text { Act } \underset{\alpha . E \stackrel{\alpha}{\rightarrow} E}{ }, \\
\operatorname{Sum}_{j} \frac{E_{j} \stackrel{\alpha}{\rightarrow} E_{j}^{\prime}}{\sum_{i \in I} E_{i} \stackrel{\alpha}{\rightarrow} E_{j}^{\prime}} \quad(j \in I),
\end{gathered}
$$

$$
\begin{aligned}
& \mathrm{Com}_{1} \frac{E \stackrel{\alpha}{\rightarrow} E^{\prime}}{E\left|F \stackrel{\alpha}{\rightarrow} E^{\prime}\right| F}, \\
& \mathrm{Com}_{2} \frac{F \stackrel{\alpha}{\rightarrow} F^{\prime}}{E\left|F \stackrel{\alpha}{\rightarrow} F^{\prime}\right| F}, \\
& \mathrm{Com}_{3} \frac{E \stackrel{l}{\rightarrow} E^{\prime} F \stackrel{\bar{l}}{\rightarrow} F^{\prime}}{E\left|F \stackrel{\tau}{\rightarrow} E^{\prime}\right| F^{\prime}}, \\
& \operatorname{Res} \frac{E \stackrel{\alpha}{\rightarrow} E^{\prime}}{E \backslash L \stackrel{\alpha}{\rightarrow} E^{\prime} \backslash L} \quad(\alpha, \bar{\alpha} \notin L), \\
& \operatorname{Rel} \frac{E \stackrel{\alpha}{\rightarrow} E^{\prime}}{E[f] \stackrel{\alpha}{\rightarrow} E^{\prime}[f]}, \\
& \operatorname{Con} \frac{P \stackrel{\alpha}{\rightarrow} P^{\prime}}{A \stackrel{\alpha}{\rightarrow} P^{\prime}} \quad(A \stackrel{\text { def }}{=} P) .
\end{aligned}
$$

Transitions with strings of labels may be defined in a natural way. If $t=\alpha_{1}, \ldots, \alpha_{n} \in \mathrm{Act}^{*}=\bigcup_{n=0}^{\infty} \mathrm{Act}^{n}$, then we write $E \stackrel{t}{\rightarrow} E^{\prime}$ provided that $E \stackrel{\alpha_{1}}{\longrightarrow} E_{1} \stackrel{\alpha_{2}}{\longrightarrow} \cdots \stackrel{\alpha_{n-1}}{\longrightarrow} E_{n-1} \stackrel{\alpha_{n}}{\longrightarrow} E^{\prime}$ for some $E_{1}, \ldots, E_{n-1} \in \partial$. In this case, we call $t$ an action sequence of $E$ and $E^{\prime}$ is a $t$-derivative of $E$. If for some $t \epsilon$ Act ${ }^{*}, E^{\prime}$ is a $t$-derivative of $E$, then $E^{\prime}$ is called a derivative of E.

In the subsequent sections, we mainly consider the restriction $\left(\mathscr{P}, \operatorname{Act},\left\{\left.\stackrel{\alpha}{\rightarrow}\right|_{\mathscr{P}}: \alpha \in \operatorname{Act}\right\}\right)$ of $(\partial, \operatorname{Act},\{\stackrel{\alpha}{\rightarrow}: \alpha \epsilon$ Act $\})$ on $\mathscr{P}$, where, for each $\alpha \in$ Act, $\left.\stackrel{\alpha}{\rightarrow}\right|_{\mathscr{P}}=\stackrel{\alpha}{\rightarrow} \cap(\mathscr{P} \times \mathscr{P})$ is restriction of $\stackrel{\alpha}{\rightarrow}$ on $\mathscr{P}$. For simplicity, we always write $\stackrel{\alpha}{\rightarrow}$ for $\left.\stackrel{\alpha}{\rightarrow}\right|_{\mathscr{P}}$.

For example, suppose that a vending machine that sells CocaCola can be described as an expression of CCS:

$$
P=1 \text { d. CocaCola.collect } . P \text {. }
$$

Its behavior can be expressed as a transition diagram as in Figure 1.

2.2. Parameterized Bisimulation. The definition of environment must be introduced because the motivation of parameterized bisimulation is to parameterize the bisimulation equivalence with a special type of information about context called environment. Similar to the assumption that a process may change after performing an action, the assumption that an environment may change after consuming an action is reasonable. Thus environments and their behaviors can be described by labeled transition system $\varepsilon=($ Env, Act, $\Rightarrow)$, where Env is the set of environments, Act is the set of actions (identical to the set of actions used in the transition system of process), and $\Rightarrow$ is a subset of Env $\times$ Act $\times$ Env called consumption relation. $e \stackrel{\alpha}{\Rightarrow} e^{\prime}$ means that " $e$ may consume the action $\alpha$ and in doing so become the environment $e^{\prime}$." 


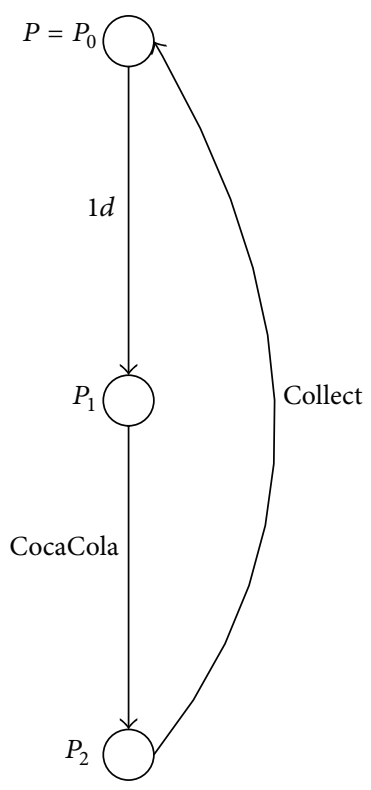

Figure 1: An example of CCS.

At this point, let us review the parameterized bisimulation equivalence. First, we recall the bisimulation equivalence without environment.

Definition 3 (bisimulation [24]). Bisimulation $R$ is a binary relation on $\mathscr{P}$ such that whenever PRQ and $\alpha \in$ Act, then

(1) $P \stackrel{\alpha}{\rightarrow} P^{\prime} \Rightarrow \exists Q^{\prime}$ such that $Q \stackrel{\alpha}{\rightarrow} Q^{\prime}$ and $P^{\prime} R Q^{\prime}$,

(2) $Q \stackrel{\alpha}{\rightarrow} Q^{\prime} \Rightarrow \exists P^{\prime}$ such that $P \stackrel{\alpha}{\rightarrow} P^{\prime}$ and $P^{\prime} R Q^{\prime}$.

Two processes, $P$ and $Q$, are considered bisimulation if and only if bisimulation $R$ exists and satisfies $(P, Q) \in R$.

Definition 4 ( $\varepsilon$-parameterized bisimulation [24]). Let $\varepsilon=$ (Env, Act, $\Rightarrow$ ) be a transition system of environments. Then an $\varepsilon$-parameterized bisimulation, $R$, is an Env-indexed family of binary relations, $R_{e} \subseteq \mathscr{P} \times \mathscr{P}$ for $e \in$ Env, s.t. whenever $P R_{e} Q$ and $e \stackrel{\alpha}{\Rightarrow} f$, then we have the following:

(1) If $P \stackrel{\alpha}{\rightarrow} P^{\prime}$, then there exists $Q^{\prime}$, s.t. $Q \stackrel{\alpha}{\rightarrow} Q^{\prime}$ and $P^{\prime} R_{f} Q^{\prime}$.

(2) If $Q \stackrel{\alpha}{\rightarrow} Q^{\prime}$, then there exists $P^{\prime}$, s.t. $P \stackrel{\alpha}{\rightarrow} P^{\prime}$ and $P^{\prime} R_{f} Q^{\prime}$.

Two processes, $P$ and $Q$, are said to be bisimulation equivalence in the environment $e \in$ Env if and only if $\varepsilon$ parameterized bisimulation $R$ exists, such that $P R_{e} Q$, which is denoted by $P \sim_{e} Q$.

Example 5 (see [24]). Let $\varepsilon, P$, and $Q$ be presented by Figure 2. The Env-indexed family $R$ is shown as follows:

$$
\begin{aligned}
& R_{e_{0}}=\left\{\left(P_{0}, Q_{0}\right)\right\} . \\
& R_{e_{1}}=\left\{\left(P_{1}, Q_{1}\right),\left(P_{2}, Q_{2}\right),\left(P_{1}, Q_{5}\right)\right\} .
\end{aligned}
$$
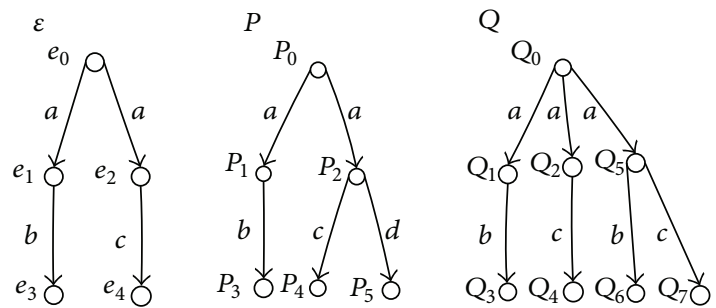

FIGURE 2: $P_{0}$ and $Q_{0}$ are parameterized bisimulation on environment $e_{0}$.

$$
\begin{aligned}
& R_{e_{2}}=\left\{\left(P_{2}, Q_{2}\right),\left(P_{1}, Q_{1}\right),\left(P_{2}, Q_{5}\right)\right\} . \\
& R_{e_{3}}=\left\{\left(P_{3}, Q_{6}\right),\left(P_{3}, Q_{3}\right)\right\} . \\
& R_{e_{4}}=\left\{\left(P_{4}, Q_{7}\right),\left(P_{4}, Q_{4}\right)\right\} .
\end{aligned}
$$

We can prove that $R$ is a parameterized bisimulation. Thus, $P_{0} \sim e_{0} Q_{0}, P_{0} \nsim Q_{0}$. Therefore, $P_{0}$ can accept the action $a, Q_{0}$ can also accept the same action, and their next states have the relation $R_{e_{1}}$ when the environment $e_{0}$ can consume the action $a$ to the next environment $e_{1}$. By contrast, if $Q_{0}$ can accept the action $a$, then $P_{0}$ can also accept the same action, and their next states have the relation $R_{e_{1}}$. Similarly, $P_{0}$ and $Q_{0}$ have the same behavior when environment $e_{0}$ can consume action $a$ to the next environment $e_{2}$. Thus, $P_{0} \sim e_{0} Q_{0}$. However, according to Definition 3, we can observe that $P_{0}+Q_{0}$.

Although $\left(P_{2}, Q_{2}\right) \in R_{e_{1}}, P_{2}$ and $Q_{2}$ will never be considered when $P_{2}$ and $Q_{2}$ are executed in environment $e_{1}$. The reason is that when $e_{1}$ consumes the action $b$ to the environment $e_{3}, P_{2}$ and $Q_{2}$ cannot execute the action $b$ to the next state.

Proposition 6 (see [24]). For alle $\in$ Env and for all $P, Q \in \mathscr{P}$, $P \sim Q$ implies that $P \sim{ }_{e} Q$.

This proposition indicates that parameterized bisimulation equivalence generalizes bisimulation equivalence.

\section{Approximate Parameterized Bisimulation}

For the approximate version of parameterized bisimulation, we present the definition of parameterized bisimulation index over the environment that indicates the degree to which a binary relation is parameterized bisimulation. We also generalize some algebraic properties of parameterized bisimulation.

Definition 7 (metric space [30]). Let $M$ be a nonempty set. $\rho$ is a mapping from $M \times M$ into $[0, \infty]$. Then the pair $(M, \rho)$ is called a metric space if the following conditions are satisfied:

(1) $\rho(x, y)=0$ if and only if $x=y$.

(2) $\rho(x, y)=\rho(y, x)$.

(3) $\rho(x, z) \leq \rho(x, y)+\rho(y, z)$ for any $x, y, z \in M$.

If (1) is weakened by $(1)^{\prime}: \rho(x, x)=0$ for each $x \in M$, then $\rho$ is called a pseudometric. If (3) is strengthened by $(3)^{\prime}$ : 
$\rho(x, z) \leq \max \{\rho(x, y), \rho(y, z)\}$ for any $x, y, z \in M$, then $\rho$ is called an ultrametric.

Let $\rho$ be a metric on $\mathscr{A}$. As expected, we can extend $\rho$ to a mapping from Act $\times$ Act to $[0, \infty]$, which is denoted by $\rho_{\text {act }}$ in the following way: for any $a, b \in \mathscr{A}$,

(1) $\rho_{\text {act }}(\tau, \tau)=0$,

(2) $\rho_{\text {act }}(a, b)=\rho_{\text {act }}(\bar{a}, \bar{b})=\rho(a, b)$,

(3) $\rho_{\mathrm{act}}(\tau, b)=\rho_{\mathrm{act}}(a, \tau)=\rho_{\mathrm{act}}(\tau, \bar{b})=\rho_{\mathrm{act}}(\bar{a}, \tau)=$ $\rho_{\text {act }}(a, \bar{b})=\rho_{\text {act }}(\bar{a}, b)=\infty$.

$\rho_{\text {act }}$ is clearly a metric on Act. In addition, $\rho_{\text {act }}$ is also an ultrametric provided that $\rho$ is an ultrametric. For simplicity, we always write $\rho$ for $\rho_{\text {act }}$. Then the numerical generalization of Definition 4 will be defined. Similar to the parameterized bisimulation, the following assumption is obtained: if $e_{1}$ can consume an action to $e_{2}, P$ and $Q$ have the relation $R_{e_{1}}$, but no transitions exist that can make $P$ and $Q$ execute certain actions to obtain some states that are included in $R_{e_{2}}$, then $P$ and $Q$ will never be considered when $P$ and $Q$ are executed in $e_{1}$.

Definition 8. Let $\left(\mathscr{P}\right.$, Act, $\left\{\left.\stackrel{\alpha}{\rightarrow}\right|_{\mathscr{P}}: \alpha \in\right.$ Act $\left.\}\right)$ be a labeled transition system and let $\rho$ be a metric on Act. $\varepsilon=($ Env, Act, $\Rightarrow)$ is an environment transition system. $R$ is an Env-indexed family of binary relations $R_{e} \subseteq \mathscr{P} \times \mathscr{P}$. For $e \in$ Env, $e \stackrel{\alpha}{\Rightarrow} e^{\prime}$, and $(P, Q) \in R_{e}$, we define that

$$
b_{R_{\left(e, e e^{\prime} ; \alpha\right)}}(P, Q)=\max \left\{b_{R_{\left(e, e^{\prime} ; \alpha\right)}}^{\neq}(P, Q), b_{R_{\left(e, e^{\prime} ; \alpha\right)}^{=}}^{=}(P, Q)\right\},
$$

where

$$
\begin{aligned}
& b_{R_{\left(e, e^{\prime} ; \alpha\right)}}^{\neq}(P, Q)=\sup \left\{b_{R_{\left(e, e^{\prime} ; \alpha\right)}}^{\neq}\left(P, Q^{\prime} ; t\right): Q^{\prime} \in \mathscr{P} \text { and } t\right. \\
& \left.\in \text { Act with } Q \stackrel{t}{\longrightarrow} Q^{\prime} \text {, if } t \neq \alpha\right\}, \\
& b_{R_{\left(e, e^{\prime} ; \alpha\right)}^{=}}^{=}(P, Q)=\sup \left\{b_{R_{\left(e, e^{\prime} ; \alpha\right)}^{=}}^{=}\left(P, Q^{\prime} ; t\right): Q^{\prime} \in \mathscr{P} \text { and } t\right. \\
& \left.\in \text { Act with } Q \stackrel{t}{\longrightarrow} Q^{\prime} \text { if } t=\alpha\right\}, \\
& b_{R_{\left(e, e^{\prime} ; \alpha\right)}^{*}}^{\neq}\left(P, Q^{\prime} ; t\right)=\inf \{\rho(t, u): u
\end{aligned}
$$

$\in$ Act and there is $P^{\prime} \in \mathscr{P}$ such that $P$

$$
\stackrel{u}{\longrightarrow} P^{\prime} \text { and } \rho(u, \alpha)-\rho(t, \alpha) \leq 0 \text {, and } Q^{\prime} R_{e^{\prime}} P^{\prime} \text {, if } t
$$

$$
\neq \alpha\}
$$

$$
b_{R_{\left(e, e^{\prime} ; \alpha\right)}}^{=}\left(P, Q^{\prime} ; t\right)=\inf \{\rho(t, u): u
$$

$\in$ Act and there is $P^{\prime} \in \mathscr{P}$ such that $P$

$$
\left.\stackrel{u}{\longrightarrow} P^{\prime} \text {, and } Q^{\prime} R_{e^{\prime}} P^{\prime} \text {, if } t=\alpha\right\} \text {. }
$$

We call $b_{R_{\left(e, e^{\prime} ; \alpha\right)}}(P, Q)$ an index in which $P$ simulates $Q$ on the transition $e \stackrel{\alpha}{\Rightarrow} e^{\prime}$.

Definition 9. Let $\left(\mathscr{P}\right.$, Act, $\left\{\left.\stackrel{\alpha}{\rightarrow}\right|_{\mathscr{P}}: \alpha \in\right.$ Act $\left.\}\right)$ be a labeled transition system and let $\rho$ be a metric on Act. $\varepsilon=($ Env, Act,$\Rightarrow)$ is an environment transition system. $R$ is an Env-indexed family of binary relations $R_{e} \subseteq \mathscr{P} \times \mathscr{P}$. If $e \in$ Env, such that

$$
b_{R_{e}}=\sup \left\{b_{R_{\left(e, e^{\prime} ; \alpha\right)}}: e \stackrel{\alpha}{\Longrightarrow} e^{\prime}\right\}
$$

where

$$
\begin{aligned}
& b_{R_{\left(e, e^{\prime} ; \alpha\right)}} \\
& \quad=\sup \left\{\max \left\{b_{R_{\left(e, e^{\prime} ; \alpha\right)}}(P, Q), b_{R_{\left(e, e^{\prime} ; \alpha\right)}^{-1}}(Q, P): P R_{e} Q\right\}\right\},
\end{aligned}
$$

then $b_{R_{e}}$ is called parameterized bisimulation index of $R$ over environment $e$.

As expected, if $Q, Q^{\prime}, P \in \mathscr{P}, t \in$ Act and $e \stackrel{\alpha}{\Rightarrow} e^{\prime}$ given, and $P R_{e} Q$ and $Q \stackrel{t}{\rightarrow} Q^{\prime}$, then $b_{R_{\left(e, e^{\prime} ; \alpha\right)}^{=}}^{=}\left(P, Q^{\prime} ; t\right)$ and $b_{R_{\left(e, e^{\prime} ; \alpha\right)}^{\neq}}^{\neq}\left(P, Q^{\prime} ; t\right)$ are the infimum of distances between transitions $t$ and $u$ where $t$ and $u$ are close to $\alpha$. From this point, Definition 9 is clearly a numerical counterpart of Definition 4 and $b_{R_{e}}$ expresses the degree to which $R_{e}$ is parameterized bisimulation. We should indicate that the smaller the value of $b_{R_{e}}$, the higher the degree to which $R_{e}$ is a bisimulation. We can obtain the conclusion that, for every $e \in \mathrm{Env}, b_{R_{e}}=0$ when $R$ is parameterized bisimulation.

Proposition 10. (1) $R$ is parameterized bisimulation if and only if, for every e $\in E n v, b_{R_{e}}=0$. In particular, $b_{I d_{R_{e}}}=0$, where $I d_{R_{e}}$ is the identical relation between processes.

(2) For all e $\in$ Env, $b_{R_{e}^{-1}}=b_{R_{e}}$.

(3) For all $e \in E n v, b_{R_{e}^{1} \circ R_{e}^{2}} \leq b_{R_{e}^{1}}+b_{R_{e}^{2}}$. In particular, if $\rho$ is an ultrametric, then $b_{R_{e}^{1} \circ R_{e}^{2}} \leq \max \left\{b_{R_{e}^{1}}, b_{R_{e}^{2}}\right\}$.

(4) For all $e \in E n v, b_{i \in I} R_{e}^{i} \leq \sup _{i \in I} b_{R_{e}^{i}}$.

Proof. (1) and (2) are direct from Definition 9.

(3) If $b_{R_{e}^{1}}=\infty$ or $b_{R_{e}^{2}}=\infty$, then it is clear. At this point, suppose that $b_{R_{e}^{1}}<\infty$ and $b_{R_{e}^{2}}<\infty$. Sequences $\left\{\lambda_{1 n}\right\}$ and $\left\{\lambda_{2 n}\right\}$ exist, such that $b_{R_{e}^{1}}<\lambda_{1 n}$ and $b_{R_{e}^{2}}<\lambda_{2 n}(n=1,2, \ldots)$, $\lim _{n \rightarrow \infty} \lambda_{1 n}=b_{R_{e}^{1}}, \lim _{n \rightarrow \infty} \lambda_{2 n}=b_{R_{e}^{2}}$, for any $e \stackrel{\alpha}{\Rightarrow} e^{\prime}$, $b_{R_{\left(e, e^{\prime} ; \alpha\right)}^{1}} \leq \lambda_{1 n}$, and $b_{R_{\left(e, e^{2} ; \alpha\right)}^{2}} \leq \lambda_{2 n}$.

For any $P, W \in \mathscr{P}$, if $P\left(R_{e}^{1} \circ R_{e}^{2}\right) W$, then there exists $Q \in \mathscr{P}$ with $P R_{e}^{1} Q$ and $Q R_{e}^{2} W$. For any $P^{\prime} \in \mathscr{P}$ and $t_{1} \in$ Act, $P \stackrel{t_{1}}{\rightarrow}$ $P^{\prime}$. If $t_{1}=\alpha$, then $b_{R_{\left(e, e^{\prime} ; \alpha\right)}^{=}}^{=}<\lambda_{1 n}$ leads to the idea that there exist $Q^{\prime} \in \mathscr{P}$ and $t_{2} \in$ Act such that $Q \stackrel{t_{2}}{\rightarrow} Q^{\prime}, P^{\prime} R_{e^{\prime}}^{1} Q^{\prime}$, and $\rho\left(t_{1}, t_{2}\right) \leq \lambda_{1 n}$. At the same time, if $t_{1} \neq \alpha, b_{R_{\left(e, e^{\prime} ; \alpha\right)}^{\neq}}^{\neq}<\lambda_{1 n}$ also leads to the idea that there exist $Q^{\prime} \in \mathscr{P}$ and $t_{2} \in$ Act such that $Q \stackrel{t_{2}}{\rightarrow} Q^{\prime}$ with $\rho\left(t_{2}, \alpha\right) \leq \rho\left(t_{1}, \alpha\right), P^{\prime} R_{e^{\prime}}^{1} Q^{\prime}$, and $\rho\left(t_{1}, t_{2}\right) \leq \lambda_{1 n}$. 

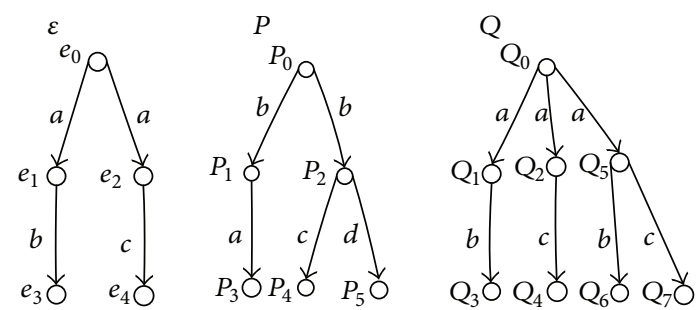

FIgURE 3: An example of parameterized bisimulation index.

Furthermore, $b_{R_{\left(e, e^{\prime} ; \alpha\right)}^{2}}<\lambda_{2 n}$ leads to the following: if $t_{1}=$ $\alpha, b_{R_{\left(e, e^{\prime} ; \alpha\right)}^{=}}^{=}\left(W, Q^{\prime} ; t_{2}\right) \leq \lambda_{2 n}$. Thus, $W^{\prime} \in \mathscr{P}$ and $t_{3} \in$ Act exist, such that $W \stackrel{t_{3}}{\rightarrow} W^{\prime}$ with $\rho\left(t_{2}, t_{3}\right)<\lambda_{2 n}$ and $Q^{\prime} R_{e^{\prime}}^{2} W^{\prime}$. Moreover, if $t_{1} \neq \alpha, b_{R_{\left(e, e^{\prime} ; \alpha\right)}^{\neq}}^{\neq}\left(W, Q^{\prime} ; t_{2}\right) \leq \lambda_{2 n}$. We can obtain $W^{\prime} \in \mathscr{P}$ and $t_{3} \in$ Act such that $W \stackrel{t_{3}}{\rightarrow} W^{\prime}$ with $\rho\left(t_{3}, \alpha\right) \leq$ $\rho\left(t_{2}, \alpha\right), Q^{\prime} R_{e^{\prime}}^{2} W^{\prime}$, and $\rho\left(t_{2}, t_{3}\right)<\lambda_{2 n}$. Therefore, $P^{\prime}\left(R_{e^{\prime}}^{1}\right.$ 。 $\left.R_{e^{\prime}}^{2}\right) W^{\prime}$ with $\rho\left(t_{3}, \alpha\right) \leq \rho\left(t_{1}, \alpha\right)$ and $\rho\left(t_{1}, t_{3}\right) \leq \rho\left(t_{1}, t_{2}\right)+$ $\rho\left(t_{2}, t_{3}\right)<\lambda_{1 n}+\lambda_{2 n} \cdot b_{R_{e}^{1} \circ R_{e}^{2}} \leq \lambda_{1 n}+\lambda_{2 n}(n=1,2, \ldots)$. And $b_{R_{e}^{1} \circ R_{e}^{2}} \leq \lim _{n \rightarrow \infty}\left(\lambda_{1 n}+\lambda_{2 n}\right)=\lim _{n \rightarrow \infty} \lambda_{1 n}+\lim _{n \rightarrow \infty} \lambda_{2 n}=$ $b_{R_{e}^{1}}+b_{R_{e}^{2}}$.

(4) is similar to (3).

(1) in Proposition 10 indicates that, for any $e \in$ Env, the parameterized bisimulation index is 0 , which is the least value of the bisimulation index over the environment e. (2) states that, for any environment $e \in$ Env, the bisimulation index of relation $R_{e}$ and the bisimulation index of its inverse are the same. (3) means that, for any environment $e \in$ Env, the bisimulation index of the composition of two relations is not greater than the sum of the bisimulation indexes of the relation. If the presumed metric on actions is an ultrametric, then it does not exceed even the greatest of the bisimulation indexes of the factor relations. Finally, (4) means that if the degree to which $R_{e}^{i}$ is a bisimulation is not less than some values for all $i \in I$, then the degree to which $\bigcup_{i \in I} R_{e}^{i}$ is a bisimulation is also not less than that value.

Example 11. Let $\varepsilon$ and $P, Q$ be illustrated by Figure 3. The Envindexed family $R$ is shown as follows:

$$
\begin{aligned}
& R_{e_{0}}=\left\{\left(P_{0}, Q_{0}\right)\right\}, \\
& R_{e_{1}}=\left\{\left(P_{1}, Q_{1}\right),\left(P_{2}, Q_{2}\right),\left(P_{1}, Q_{5}\right)\right\}, \\
& R_{e_{2}}=\left\{\left(P_{2}, Q_{2}\right),\left(P_{1}, Q_{1}\right),\left(P_{2}, Q_{5}\right)\right\}, \\
& R_{e_{3}}=\left\{\left(P_{3}, Q_{6}\right),\left(P_{3}, Q_{3}\right)\right\}, \\
& R_{e_{4}}=\left\{\left(P_{4}, Q_{7}\right),\left(P_{4}, Q_{4}\right)\right\} .
\end{aligned}
$$

Let the metric on Act be defined as

$$
\begin{aligned}
& \rho(a, b)=\rho(b, a)=0.1, \\
& \rho(a, c)=\rho(c, a)=0.6, \\
& \rho(a, d)=\rho(d, a)=0.6
\end{aligned}
$$

$$
\begin{aligned}
& \rho(b, c)=\rho(c, b)=0.7, \\
& \rho(b, d)=\rho(d, b)=0.4, \\
& \rho(c, d)=\rho(d, c)=0.8 .
\end{aligned}
$$

Then, we can obtain that $b_{R_{e_{0}}}=0.1, b_{R_{e_{1}}}=0.1, b_{R_{e_{2}}}=0$, and $b_{R_{e_{3}}}=b_{R_{e_{4}}}=0$.

In fact, given that $e_{0} \stackrel{a}{\Rightarrow} e_{1}$, we should first compute $b_{R_{\left(e_{0}, e_{1} ; a\right)}}$ when $b_{R_{e_{0}}}$ is computed. Moreover, $\left(P_{0}, Q_{0}\right) \in R_{e_{0}}$ leads to the idea that $b_{R_{\left(e_{0}, e_{1} ; a\right)}}\left(P_{0}, Q_{0}\right)$ and $b_{R_{\left(e_{0}, e_{1} ; a\right)}^{-1}}^{-}\left(Q_{0}, P_{0}\right)$ should be gained. Since $Q_{0} \stackrel{a}{\rightarrow} Q_{1}$, we only need to compute $b_{R_{\left(e_{0}, e_{1} ; a\right)}}^{=}\left(P, Q_{1} ; t=a\right)$. By $P_{0} \stackrel{b}{\rightarrow} P_{1}$ and $\left(P_{1}, Q_{1}\right) \in R_{e_{1}}$, the metric $\rho(b, a)=0.1$ can be obtained according to Definition 9 . Meanwhile, $P_{0} \stackrel{b}{\rightarrow} P_{2}$, but $\left(P_{2}, Q_{1}\right) \notin R_{e_{1}}$, so this transition should not be considered. Thus, we obtain $b_{R_{\left(e_{0}, e_{1} ; a\right)}^{=}}^{=}\left(P, Q_{1} ; t=\right.$ $a)=0.1$. Similarly, we get that $b_{R_{\left(e_{0}, e_{1} ; a\right)}^{=}}^{=}\left(P, Q_{2} ; t=a\right)=0.1$, $b_{R_{\left(e_{0}, e_{1} ; a\right)}}^{=}\left(P, Q_{3} ; t=a\right)=0.1$. Thus $b_{R_{\left(e_{0}, e_{1} ; a\right)}}^{=}\left(P_{0}, Q_{0}\right)=0.1$.

By contrast, we only need to compute $b_{R_{\left(e_{0}, e_{1} ; a\right)}^{-1}}^{\neq}\left(Q_{0}, P_{0}\right)$ when $P_{0} \stackrel{b}{\rightarrow} P_{1}$ because $b \neq a$. Furthermore, we should obtain $b_{R_{\left(e_{0}, e_{1} ; a\right)}^{\prime}}^{\neq}\left(Q_{0}, P_{1} ; b\right)$. Since $\rho(a, b)=0.1$, we only choose the transition of $Q_{0}$ which satisfies the metric between $a$ and the action $u$ of $Q_{0}$ executing less than $\rho(b, a)$. All transitions of $Q_{0}$ both satisfy the metric between $a$ and the actions that $Q_{0}$ can perform is less than and equal to 0.1. However, $\left(P_{1}, Q_{2}\right) \notin R_{e_{1}}$. Thus, we can obtain $b_{R_{\left(e_{0}, e_{1} ; a\right)}^{*}}^{\neq}\left(Q_{0}, P_{1} ; b\right)=0.1$. Similarly, we can gain $b_{R_{\left(e_{0}, e_{1} ; a\right)}^{-1}}^{\neq}\left(Q_{0}, P_{2} ; b\right)=0.1$. Thus, $b_{R_{\left(e_{0}, e_{1} ; a\right)}^{-1}}^{\neq}\left(Q_{0}, P_{0}\right)=0.1$. Moreover, $b_{R_{\left(e_{0}, e_{1} ; a\right)}}=0.1$. Furthermore, we also get $b_{R_{\left(e_{0}, e_{2} ; a\right)}}=$ 0.1 when $e_{0} \stackrel{a}{\Rightarrow} e_{2}$. Therefore, $b_{R_{e_{0}}}=0.1$.

In particular, $\left(P_{2}, Q_{2}\right)$ will never be considered when we compute $b_{R_{e_{1}}}$ and $e_{1} \stackrel{b}{\Rightarrow} e_{3}$. The reason is that the next states of $\left(P_{2}, Q_{2}\right)$ are not in $R_{e_{3}}$. The other results can be obtained in the same way.

Proposition 12. Let $\varepsilon=(E n v, A c t, \Rightarrow)$ be an environment transition system. If $R$ is a strong bisimulation relation, then, for every e $\in E n v, b_{R}=b_{R_{e}}$, where $b_{R}$ is the bisimulation index defined in [7].

Definition 13. Let $\sigma=(\mathscr{P}$, Act, $\{\stackrel{\alpha}{\rightarrow}: \alpha \in$ Act $\})$ be a labeled transition system. $\varepsilon=($ Env, Act, $\Rightarrow)$ is an environment transition system. $R$ is an Env-indexed family of binary relations on $\mathscr{P} \times \mathscr{P}$; that is, $R_{e} \subseteq \mathscr{P} \times \mathscr{P}$ for $e \in$ Env, $\lambda \in[0, \infty)$. If $b_{R_{e}} \leq \lambda$, then $R_{e}$ is called a $\lambda$-parameterized bisimulation over the environment $e$.

If $R$ is an Env-indexed family of binary relations on $\mathscr{P} \times \mathscr{P}$, $e \in$ Env, then $R_{e}$ is clearly an $\infty$-parameterized bisimulation. If $\lambda_{1} \leq \lambda_{2}$ and $R_{e}$ is $\lambda_{1}$-parameterized bisimulation, then $R_{e}$ is also a $\lambda_{2}$-parameterized bisimulation. Moreover, if $R_{e}$ 

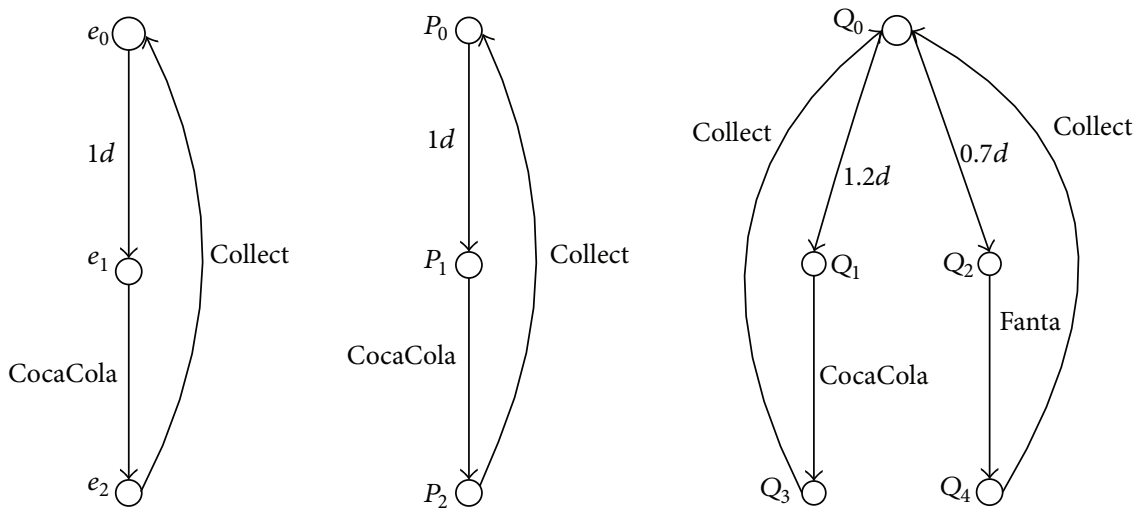

FIgURE 4: $P_{0}$ and $Q_{0}$ are 0.3 -bisimilar on the environment $e_{0}$.

is $\lambda_{i}$-parameterized bisimulation $(i \in I)$, then $R_{e}$ is a inf ${ }_{i \in I} \lambda_{i^{-}}$ parameterized bisimulation.

Corollary 14. Let $\sigma=(\mathscr{P}$, Act, $\{\stackrel{\alpha}{\rightarrow}: \alpha \in$ Act $\})$ be a labeled transition system. $\varepsilon=(E n v, A c t, \Rightarrow)$ is an environment transition system. $R$ is an Env-indexed family of binary relations on $\mathscr{P} \times \mathscr{P}$.

(1) If $R$ is a parameterized bisimulation, then, for any environment $e \in E n v, R_{e}$ is a 0-parameterized bisimulation.

(2) If $R$ is a parameterized bisimulation, $e \in E n v, R_{e}$ is a $\lambda$-parameterized bisimulation, if and only if so is $R_{e}^{-1}$.

(3) If, for e $\in E n v, R_{e}^{i}$ is a $\lambda_{i}$-parameterized bisimulation $(i=1,2)$, then $R_{e}^{1} \circ R_{e}^{2}$ is a $\lambda_{1}+\lambda_{2}$-parameterized bisimulation. In particular, if $\rho$ is an ultrametric and $R_{1}$ and $R_{2}$ are all $\lambda$-bisimulation, so is $R_{1} \circ R_{2}$.

(4) If, for e $\in E n v, R_{e}^{i}$ is a $\lambda$-bisimulation ( $i \in I$ ), so is $\bigcup_{i \in I} R_{e}^{i}$.

Using the concept of $\lambda$-bisimulation, we can define the notion of $\lambda$-parameterized bisimulation in the usual way over the environment $e$.

Definition 15. Let $R$ be an Env-indexed family of binary relations on $\mathscr{P} \times \mathscr{P}$. For any $\lambda \in[0, \infty)$, we define $\lambda$ bisimulation over the environment $e$ as

$$
\sim_{e}^{\lambda}=\bigcup\left\{R_{e} \mid\right.
$$

$R_{e}$ is a $\lambda$-parameterized bisimulation over the environment $\left.e\right\}$.

In other words, if $(P, Q) \in R_{e}$, then $P$ and $Q$ are said to be $\lambda$-bisimilar over the environment $e$ whenever $\lambda$-parameterized bisimulation $R$ exists such that $(P, Q) \in R_{e}$. If $(P, Q) \in$ $R_{e}$, e can consume some actions to $e^{\prime}$, but $P$ and $Q$ do not have any transition such that their next states are included in $R_{e^{\prime}}$, then $P$ and $Q$ will never be considered when $P$ and $Q$ are executed in the environment $e$.

Next, we illustrate Definitions 9 and 15 with the following example.
Example 16. Two vending machines are assumed to exist. They can be expressed by the following process expressions:

$$
\begin{aligned}
& P=1 d . \text { CocaCola.Collect. } P \\
& Q=(1.2 d \text {.CocaCola.Collect }+0.7 \text {. Fanta.Collect }) . Q \text {. }
\end{aligned}
$$

A person who wants to buy a cup of CocaCola can be treated as an environment of the vending machines. According to CCS, the behaviors of the person can be described as a process:

$$
\varepsilon=1 \text { d. CocaCola.Collect. } \varepsilon .
$$

The transition diagrams are described in Figure 4. Metric $\rho$ exists on the set of actions Act, where Act $=\{1 d, 1.2 d, 0.7 d$, CocaCola, Fanta, Collect $\}$. Consider $\rho(1 d, 1.2 d)=0.2, \rho(1 d$, $0.7)=0.3, \rho(1.2 d, 0.7 d)=0.5$, and $\rho$ (CocaCoca, Fanta $)=$ 0.2 . The distance between other actions is $\infty$.

The following relations can be defined:

$$
\begin{aligned}
& R_{e_{0}}=\left\{\left(P_{0}, Q_{0}\right)\right\} \\
& R_{e_{1}}=\left\{\left(P_{1}, Q_{1}\right),\left(P_{1}, Q_{2}\right)\right\} \\
& R_{e_{2}}=\left\{\left(P_{2}, Q_{3}\right),\left(P_{2}, Q_{4}\right)\right\} .
\end{aligned}
$$

We can get that $b_{R_{e_{0}}}=0.3, b_{R_{e_{1}}}=0.2, b_{R_{e_{2}}}=0$. So, $P_{0} \sim_{e_{0}}^{0.3} Q_{0}, P_{1} \sim{ }_{e_{1}}^{0.2} Q_{1}, P_{1} \sim{ }_{e_{1}}^{0.2} Q_{2}, P_{2} \sim{ }_{e_{2}}^{0} Q_{3}$, and $P_{2} \sim e_{e_{2}}^{0} Q_{4}$. The value means that when the person does not do anything, the approximate degree between two vending machines is 0.7 . When the person puts $1 d$, then the approximate degree between two vending machines is 0.8. Finally, when the person chooses the CocaCola, the distance between them is 0.

Next, we will try to prove various properties of $\lambda$-parameterized bisimulation over environment $e$.

Proposition 17. Consider

(1) $\sim_{e} \subseteq \sim_{e}^{0}$. If $\lambda_{1} \leq \lambda_{2}$, then $\sim_{e}^{\lambda_{1}} \subseteq \sim_{e}^{\lambda_{2}}$.

(2) For any $\lambda \in[0, \infty), \sim_{e}^{\lambda}$ is a $\lambda$-parameterized bisimulation and it is reflexive and symmetric; $\sim_{e}^{\lambda_{1}} \circ \sim_{e}^{\lambda_{2}}=\sim_{e}^{\lambda_{1}+\lambda_{2}}$.

Proposition 18. Let $e \in E n v . s_{1} \sim_{e}^{\lambda} s_{2}$ if and only if, for any $e \stackrel{\alpha}{\Rightarrow} e^{\prime}, b_{\left(\sim_{e}^{\lambda}\right)_{\left(e, e^{\prime} ; \alpha\right)}}\left(s_{1}, s_{2}\right) \leq \lambda$ and $b_{\left(\sim_{e}^{\lambda}\right)_{\left(e, e^{\prime} ; \alpha\right)}}\left(s_{2}, s_{1}\right) \leq \lambda$. 
Proof. If $s_{1} \sim_{e}^{\lambda} s_{2}$, then we have that $b_{\left(\sim_{e}^{\lambda}\right)_{\left(e, e^{\prime} ; \alpha\right)}}\left(s_{1}, s_{2}\right) \leq \lambda$ and $b_{\left(\sim_{e}^{\lambda}\right)_{\left(e, e^{\prime} ; \alpha\right)}}\left(s_{2}, s_{1}\right) \leq \lambda$.

Conversely, we define that $\left(s_{1}, s_{2}\right) \in R_{e}$ if and only if $b_{\left.\left(\sim_{e}^{\lambda}\right)_{(e, e} ; \alpha\right)}\left(s_{1}, s_{2}\right) \leq \lambda$ and $b_{\left(\sim_{e}^{\lambda}\right)_{\left(e, e^{\prime} ; \alpha\right)}}\left(s_{2}, s_{1}\right) \leq \lambda$ for all $e \stackrel{\alpha}{\Rightarrow} e^{\prime}$. From $b_{\left(\sim_{e}^{\lambda}\right)_{\left(e, e^{\prime} ; \alpha\right)}}\left(s_{1}, s_{2}\right) \leq \lambda$, we know that, for any $s_{1}^{\prime} \in \mathscr{P}$ and $t \in$ Act with $s_{1} \stackrel{t}{\rightarrow} s_{1}^{\prime}$, if $t=\alpha$, then, for any $n \geq 1$, there exist $s_{2}^{\prime} \in \mathscr{P}$ and $t \in$ Act such that $s_{2} \stackrel{t^{\prime}}{\rightarrow} s_{2}^{\prime}$ and $\rho\left(t, t^{\prime}\right)<\lambda+1 / n$ and $s_{1}^{\prime} \sim_{e^{\prime}}^{\lambda} s_{2}^{\prime}$; if $t \neq \alpha$, then, for any $n \geq 1$, there exist $s_{2}^{\prime} \in \mathscr{P}$ and $t \in$ Act such that $s_{2} \stackrel{t^{\prime}}{\rightarrow} s_{2}^{\prime}$ and $\rho\left(t^{\prime}, \alpha\right) \leq \rho(t, \alpha), \rho\left(t, t^{\prime}\right)<$ $\lambda+1 / n$, and $s_{1}^{\prime} \sim_{e^{\prime}}^{\lambda} s_{2}^{\prime}$. By noting that $s_{1}^{\prime} \sim_{e^{\prime}}^{\lambda} s_{2}^{\prime}$ implies $s_{1}^{\prime} R_{e^{\prime}} s_{2}^{\prime}$, we obtain $b_{R_{\left(e, e^{\prime} ; \alpha\right)}}\left(s_{1}, s_{2}\right) \leq \lambda$. Similarly, $b_{R_{\left(e, e^{\prime} ; \alpha\right)}^{-1}}\left(s_{2}, s_{1}\right) \leq \lambda$. Therefore, $R_{e}$ is a $\lambda$-parameterized bisimulation over the environment $e$, and $s_{1} \sim{ }_{e}^{\lambda} s_{2}$.

\section{Congruence of $\lambda$-Parameterized Bisimulation over Environment $e$}

In order to support hierarchic development and modular decomposition of software, it is necessary to ensure that equivalences are congruent with respect to processes combinators. It means that if two processes are equivalent, then the new processes obtained by combining the given processes are also equivalent. In this section, we will mainly discuss these substitutivity laws of $\lambda$-parameterized bisimulation under various combinators.

Definition 19 ( $\lambda$-round [7]). Let $(M, \rho)$ be a metric space; $Y \subseteq$ $M, \lambda \geq 0$. If, for any $x, y \in M, x \in Y$ and $\rho(x, y) \leq \lambda$ implies $y \in Y$, then $Y$ is said to be $\lambda$-round. If, for some $\mu>\lambda, Y$ is $\mu$-round, then $Y$ is said to be strongly $\lambda$-round.

Definition 20 (isomorphism mapping [7]). Let $(M, \rho)$ be a metric space. $f$ is a mapping from $M$ into itself. If, for any $x, y \in M, \rho(f(x), f(y))=\rho(x, y)$, then $f$ is said to be isomorphism mapping.

From the definition above, we can see that $\lambda$-round is a rigorous condition. In [7], they prove that there are only two $\lambda$-round sets in the real line when $\lambda>0$. One is the empty set and the other is the real line itself. They also show that it is not the same case as in the real line in general. For example, if $M=\bigcup_{i \in I} M_{i}$ and $\left\{M_{i}\right\}_{i \in I}$ is pairwise disjoint and $\rho(x, y)>\mu$ for any $x, y \in M$ with $x \in M_{i}$ and $y \in M_{j}(i \neq j)$, then each $M_{i}$ is $\lambda$-round for every $\lambda \leq \mu$.

We now consider the substitutivity laws of $\lambda$-parameterized bisimulation over environment $e$ under various combinators in our process calculus.

Proposition 21. Let $\varepsilon=(E n v, A c t, \Rightarrow)$ be an environment transition system; $e \in E n v$,

(1) If $P_{1} \sim{ }_{e}^{\lambda} P_{2}$ and $e^{\prime} \stackrel{\alpha}{\Rightarrow} e$, then $\alpha \cdot P_{1} \sim_{e^{\prime}}^{\lambda} \alpha \cdot P_{2}$.

(2) If $L$ is $\lambda$-round and $P_{1} \sim{ }_{e}^{\lambda} P_{2}$, then $P_{1} \backslash L \sim{ }_{e}^{\lambda} P_{2} \backslash L$.

(3) If $f$ is isomorphism mapping on Act and $P_{1} \sim_{f^{-1}(e)}^{\lambda} P_{2}$, then $P_{1}[f] \sim_{e}^{\lambda} P_{2}[f]$.
(4) If $P_{1} \sim_{e}^{\lambda} P_{2}$, then $W+P_{1} \sim_{e}^{\lambda} W+P_{2}$.

Proof. (1) We need to show $b_{\left(\sim_{e^{\prime}}^{\lambda}\right)_{\left(e^{\prime}, e ; \alpha\right)}}\left(\alpha \cdot P_{1}, \alpha \cdot P_{2}\right) \leq \lambda$ and

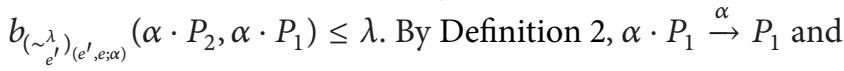
$P_{1} \sim{ }_{e}^{\lambda} P_{2}$, so there exists $P_{2}$ such that $\alpha \cdot P_{2} \stackrel{\alpha}{\rightarrow} P_{2}$ with $\rho(\alpha, \alpha) \leq$ $\rho(\alpha, \alpha)$ and $\rho(\alpha, \alpha)=0 \leq \lambda$. So $b_{\left(\sim_{e^{\prime}}^{\lambda}\right)_{\left(e^{\prime}, e ; \alpha\right)}}\left(\alpha \cdot P_{1}, \alpha \cdot P_{2}\right)=0 \leq \lambda$. Similarly, $b_{\left(\sim_{e^{\prime}}^{\lambda}\right)_{\left(e^{\prime}, e ; \alpha\right)}}\left(\alpha \cdot P_{2}, \alpha \cdot P_{1}\right) \stackrel{e^{\prime}}{=} 0 \leq \lambda$.

(2) Let $e \in$ Env, $S_{e}=\left\{\left(P_{1} \backslash L, P_{2} \backslash L\right): P_{1} \sim_{e}^{\lambda} P_{2}\right\}$. Next, we need to show $b_{S_{e}} \leq \lambda$.

Let $e \stackrel{\beta}{\Rightarrow} f$. If $P_{1} \backslash L \stackrel{\alpha}{\rightarrow} P_{1}^{\prime}$, then $\alpha, \alpha^{\prime} \notin L \cup \bar{L}, P_{1} \stackrel{\alpha}{\Rightarrow} P_{1}^{\prime \prime}$, and $P_{1}^{\prime}=P_{1}^{\prime \prime} \backslash L$. If $\alpha \neq \beta$, since $P_{1} \sim_{e}^{\lambda} P_{2}$, there exist $P_{2}^{\prime \prime} \in$ $\mathscr{P}$ and $u \in$ Act such that $P_{2} \stackrel{u}{\rightarrow} P_{2}^{\prime \prime}$ with $\rho(u, \beta) \leq \rho(\alpha, \beta)$, $\rho(\alpha, u) \leq \lambda$, and $P_{1}^{\prime \prime} \sim_{f}^{\lambda} P_{2}^{\prime \prime}$. Since $L$ is $\lambda$-round, $u, \bar{u} \neq L \cup \bar{L}$. By the transition rule, $P_{2} \backslash L \stackrel{u}{\rightarrow} P_{2}^{\prime \prime} \backslash L=P_{2}^{\prime}$ with $\rho(u, \beta) \leq \rho(\alpha, \beta)$, $\rho(\alpha, u) \leq \lambda$, and $\left(P_{1}^{\prime}, P_{2}^{\prime}\right) \in S_{f}$. If $\alpha=\beta$, then $P_{1} \sim_{e}^{\lambda} P_{2}$ leads to the idea that there exist $P_{2}^{\prime \prime} \in \mathscr{P}$ and $u \in$ Act such that $P_{2} \stackrel{u}{\rightarrow} P_{2}^{\prime \prime}$ with $\rho(\alpha, u) \leq \lambda$ and $P_{1} \sim_{f}^{\lambda} P_{2}^{\prime \prime}$. Since $L$ is $\lambda$-round, $u, \bar{u} \neq L \cup \bar{L}$. By the transition rule, $P_{2} \backslash L \stackrel{u}{\rightarrow} P_{2}^{\prime \prime} \backslash L=P_{2}^{\prime}$ with $\rho(\alpha, u) \leq \lambda$ and $\left(P_{1}^{\prime}, P_{2}^{\prime}\right) \in S_{f}$. Therefore, $b_{S_{e}} \leq \lambda$.

(3) For any $e \in$ Env, let $S_{e}=\left\{\left(P_{1}[f], P_{2}[f]\right): P_{1} \sim_{f^{-1}(e)}^{\lambda} P_{2}\right\}$. Next, we only need to show $b_{S_{e}} \leq \lambda$.

In fact, let $e \stackrel{b}{\Rightarrow} g$. If $P_{1}[f] \stackrel{\alpha}{\rightarrow} P_{1}^{\prime}$, then there exists $h \in$ Act, such that $P_{1} \stackrel{h}{\rightarrow} P_{1}^{\prime \prime}, P_{1}^{\prime \prime}[f]=P_{1}^{\prime}$, and $\alpha=f(h)$. If $\alpha \neq b$, since $P_{1} \sim_{f^{-1}(e)}^{\lambda} P_{2}, f^{-1}(e) \stackrel{f^{-1}(b)}{\Rightarrow} f^{-1}(g)$ and there exist $P_{2}^{\prime \prime} \in \mathscr{P}$ and $u \in$ Act such that $P_{2} \stackrel{u}{\rightarrow} P_{2}^{\prime \prime}$ with $\rho(h, u) \leq \lambda, \rho\left(u, f^{-1}(b)\right) \leq$ $\rho\left(h, f^{-1}(b)\right),\left(P_{1}^{\prime \prime}, P_{2}^{\prime \prime}\right) \in S_{f^{-1}(g)}$, and $P_{1}^{\prime \prime} \sim_{f^{-1}(g)}^{\lambda} P_{2}^{\prime \prime}$. By the transition rule, $P_{2}[f] \stackrel{f(u)}{\longrightarrow} P_{2}^{\prime \prime}[f]$. Let $P_{2}^{\prime}=P_{2}^{\prime \prime}[f]$ and since $f$ is isomorphism, $\rho(f(u), b)=\rho\left(f(u), f\left(f^{-1}(b)\right)\right)=$ $\rho\left(u, f^{-1}(b)\right) \leq \rho\left(h, f^{-1}(b)\right)=\rho(f(h), b)$ and $\rho(f(u), f(h))=$ $\rho(u, h) \leq \lambda$, and $\left(P_{1}^{\prime \prime}[f], P_{2}^{\prime \prime}[f]\right) \in S_{g}$.

On the other hand, if $\alpha=b, P_{1} \sim_{f^{-1}(e)}^{\lambda} P_{2}$ tells us $f^{-1}(e) \stackrel{f^{-1}(b)}{\Rightarrow} f^{-1}(g)$ and there exist $P_{2}^{\prime \prime} \in \mathscr{P}$ and $u \in$ Act such that $P_{2} \stackrel{u}{\rightarrow} P_{2}^{\prime \prime}$ with $\rho(h, u) \leq \lambda,\left(P_{1}^{\prime \prime}, P_{2}^{\prime \prime}\right) \in S_{f^{-1}(g)}$, and $P_{1}^{\prime \prime} \sim_{f^{-1}(g)}^{\lambda} P_{2}^{\prime \prime}$. By the transition rule, $P_{2}[f] \stackrel{f(u)}{\longrightarrow} P_{2}^{\prime \prime}[f]$. Let $P_{2}^{\prime}=P_{2}^{\prime \prime}[f]$ and since $f$ is isomorphism, $\rho(f(u), f(h))=$ $\rho(u, h) \leq \lambda$, and $\left(P_{1}^{\prime \prime}[f], P_{2}^{\prime \prime}[f]\right) \in S_{g}$. Therefore, $b_{S_{e}} \leq \lambda$.

(4) We need to prove that, for any $e \stackrel{b}{\Rightarrow} e^{\prime}, b_{\left(\sim_{e}^{\lambda}\right)_{\left(e, e^{\prime} ; b\right)}}(W+$ $\left.P_{1}, W+P_{2}\right) \leq \lambda$ and $b_{\left(\sim_{e}^{\lambda}\right)_{\left(e, e^{\prime} ; b\right)}}\left(W+P_{2}, W+P_{1}\right) \leq \lambda$. In fact, if $W+P_{1} \stackrel{\alpha}{\rightarrow} P^{\prime}$, then $P_{1} \stackrel{\alpha}{\rightarrow} P_{1}^{\prime}$ or $W \stackrel{\alpha}{\rightarrow} P_{1}^{\prime}$. Furthermore, if $\alpha \neq$ $b$, then $P_{1} \stackrel{\alpha}{\rightarrow} P_{1}^{\prime}$ leads to the idea that there exist $P_{2}^{\prime} \in \mathscr{P}$ and $\beta \in$ Act such that $P_{2} \stackrel{\beta}{\rightarrow} P_{2}^{\prime}$ with $\rho(\beta, b) \leq \rho(\alpha, b), \rho(\alpha, \beta) \leq \lambda$, and $\left(P_{1}^{\prime}, P_{2}^{\prime}\right) \in \sim_{f}^{\lambda}$. So, $W+P_{2} \stackrel{\beta}{\rightarrow} P_{2}^{\prime}$, and $b_{\left(\sim_{e}^{\lambda}\right)_{\left(e, e^{\prime} ; b\right)}}\left(W+P_{1}, W+\right.$ $\left.P_{2}\right) \leq \lambda$. And if $W \stackrel{\alpha}{\rightarrow} P_{1}^{\prime}$, then $W+P_{2} \stackrel{\alpha}{\rightarrow} P_{1}^{\prime}$, and $\left(P_{1}^{\prime}, P_{1}^{\prime}\right) \in \sim_{e}^{0}$, so $\left(P_{1}^{\prime}, P_{1}^{\prime}\right) \in \sim_{e}^{\lambda}$. Thus, $b_{\left.\left(\sim_{e}^{\lambda}\right)_{(e, e} ; b\right)}\left(W+P_{1}, W+P_{2}\right) \leq \lambda$. 
On the other hand, if $\alpha=b$, then $P_{1} \stackrel{\alpha}{\rightarrow} P_{1}^{\prime}$ leads to the idea that there exist $P_{2}^{\prime} \in \mathscr{P}$ and $\beta \in$ Act such that $P_{2} \stackrel{\beta}{\rightarrow} P_{2}^{\prime}$, with $\rho(\alpha, \beta) \leq \lambda$ and $\left(P_{1}^{\prime}, P_{2}^{\prime}\right) \in \sim_{f}^{\lambda}$. So, $W+P_{2} \stackrel{\beta}{\rightarrow} P_{2}^{\prime}$, and $b_{\left(\sim_{e}^{\lambda}\right)_{\left(e, e^{\prime} ; b\right)}}\left(W+P_{1}, W+P_{2}\right) \leq \lambda$. And $W \stackrel{\alpha}{\rightarrow} P_{1}^{\prime}$ tells us $W+$ $P_{2} \stackrel{\alpha}{\rightarrow} P_{1}^{\prime},\left(P_{1}^{\prime}, P_{1}^{\prime}\right) \in \sim_{e}^{0}$, and $\left(P_{1}^{\prime}, P_{1}^{\prime}\right) \in \sim_{e}^{\lambda}$. Thus, $b_{\left(\sim_{e}^{\lambda}\right)_{(e, e, j b)}}(W+$ $\left.P_{1}, W+P_{2}\right) \leq \lambda$.

Similarly, we can obtain that $b_{\left(\sim_{e}^{\lambda}\right)_{\left(e, e^{\prime} ; b\right)}}\left(W+P_{2}, W+P_{1}\right) \leq \lambda$. So, $W+P_{1} \sim{ }_{e}^{\lambda} W+P_{2}$.

\section{Parameterized Probabilistic Bisimulation}

The behaviors of some complicated software systems are often probabilistic in nature. Usually, a system with probabilistic behaviors may typically be described as a probabilistic process. van Glabbeek et al. [31] introduced three models of probabilistic processes in accordance with the relation between the occurrences of actions and transition probabilities: a reactive system, a generative system, and a stratified model. For example, Larsen and Skou [19] adopted a reactive model for probabilistic processes; Giacalone et al. [14] considered generative probabilistic processes. The probabilistic processes dealt by Smolka and Steffen [32] are in a stratified setting. These models are the extension of SCCS proposed by Milner [33]. Ying [34] proposed a new model of probabilistic process, APPA, which is a probabilistic extension of CCS. Giacalone et al. [13] relaxed the notion of probabilistic bisimulation on the class of deterministic PCCS processes, called $\epsilon$ bisimulation. Two processes can simulate each other with bound $\epsilon$ of deviation in probability. Furthermore, a natural notion of distance between deterministic PCCS processes and an accompanying metric space are proposed.

However, to show the effect of environment on the execution of software, we can extend parameterized bisimulation to the probabilistic case. Firstly, the syntax and semantics of the probabilistic processes are reviewed. As in SCCS [33], let $($ Act, $\times, 1)$ be the Abelian monoid. Intuitively, action of the form $\alpha \times \beta$ represents the simultaneous execution by a process of the actions $\alpha$ and $\beta$. It will often use juxtaposition to denote products of actions, for example, $\alpha \beta$. It is convenient to assume that $\forall \alpha \in \mathscr{A}, \exists \bar{\alpha} \in \overline{\mathscr{A}}: \alpha \times \bar{\alpha}=\bar{\alpha} \times \alpha=1$ and vice versa.

Then (Act, $\times, 1^{-}$) is an Abelian group. Let $A$ be a subset of Act such that $1 \in A$ and let $f:$ Act $\rightarrow$ Act be a monoid morphism. $X$ is assumed as a process variable. The syntax of PCCS is defined as the following definition.

Definition 22 (the syntax of PCCS [13]). The set of probabilistic process expressions is the smallest set which includes $0, X$, and the following expressions:

$$
\begin{gathered}
E::=\alpha \cdot E \mid \sum_{i \in I}\left[p_{i}\right] E_{i}, \quad p_{i} \in(0,1], \\
\sum_{i \in I} p_{i} \leq 1 \\
|E \times F| E\left\lceil A|E[f]| \text { fix }_{X} E .\right.
\end{gathered}
$$

An expression that has no free variables is called a process, and $\mathrm{Pr}$ is the set of all PCCS processes. Intuitively, 0 has no derivations, whereas $\alpha \cdot E$ performs action $\alpha$ with probability 1 and then behaves like $E$. A summation expression offers a probabilistic choice among its constituent behaviors, where $I$ is accountable, so $\sum_{i \in I} p_{i}$ is a probabilistic distribution. When $I=\emptyset$, then $0=\sum_{i \in \emptyset} E_{i}$. Product represents synchronized process composition. For the restricted expression $E \backslash A$, only actions in $A$ are visible to an observer, while morphism specifies relabeling of actions. Finally fix $_{X} E$ defines a recursive process.

Then, similar to [13], we introduce an unindexed arrow that represents the cumulative probabilistic derivation of one process by another. For $P, Q \in \operatorname{Pr}$ and $\alpha \in$ Act, we write $P \stackrel{\alpha[p]}{\longrightarrow} Q$ exactly when $p=\sum\left\{p_{i} \mid P \stackrel{\alpha\left[p_{i}\right]}{\rightarrow} i_{i} Q\right\}$, where the indices $i$ that appear on the arrows are used to distinguish different occurrences of the same probabilistic derivation. For $P \in \operatorname{Pr}, S \subseteq \operatorname{Pr}$, and $\alpha \in$ Act, we write $P \stackrel{\alpha[p]}{\longrightarrow} S$ exactly when $p=\sum_{\mathrm{Q} \in S}\{q \mid P \stackrel{\alpha[q]}{\longrightarrow} Q\}$.

Let DPr be the class of deterministic PCCS processes; that is, if $P \in \mathrm{DPr}$, then, for any $\alpha \in$ Act, $P$ has at most one probabilistic derivation of type $\alpha$. Then, the operational semantics of deterministic PCCS process can be described as follows.

Definition 23 (see [13]). The structure operational semantics of deterministic PCCS process based on probabilistic derivation is given as a set of inference rules, in the style of Plotkin:

$$
\begin{aligned}
& \text { Act } \alpha \cdot E \stackrel{\alpha[1]}{\longrightarrow} E, \\
& \operatorname{Sum}_{j} E_{j} \stackrel{\alpha[q]}{\longrightarrow} E^{\prime} \Longrightarrow \sum_{i \in I}\left[p_{i}\right] E_{i} \\
& \stackrel{\alpha\left[p_{j} \cdot q\right]}{\longrightarrow} E^{\prime}(j \in I), \\
& \text { Pro } E \stackrel{\alpha[p]}{\longrightarrow} E^{\prime}, \\
& F \stackrel{\beta[q]}{\longrightarrow} F^{\prime} \Longrightarrow E \times F \stackrel{\alpha \beta[p \cdot q]}{\longrightarrow} E^{\prime} \times F^{\prime}, \\
& \operatorname{Res} E \stackrel{\alpha[p]}{\longrightarrow} E^{\prime} \Longrightarrow E\left\lceil A \stackrel{\alpha[p / v(E, A)]}{\longrightarrow} E^{\prime}\right. \\
& \text { 「 } A(\alpha \in A) \text {, where } \\
& v(E, A)=\sum\left\{\left|p_{i}\right| E \stackrel{\alpha\left[p_{i}\right]}{\longrightarrow} E^{\prime}, \alpha \in A \mid\right\}, \\
& \operatorname{Rel} E \stackrel{\alpha[p]}{\longrightarrow} E^{\prime} \Longrightarrow E[f] \stackrel{f(\alpha)[p]}{\longrightarrow} E^{\prime}[f], \\
& \operatorname{Con} E\left\{\frac{\text { fix }_{X} E}{X}\right\} \stackrel{\alpha[p]}{\longrightarrow} E^{\prime} \Longrightarrow \operatorname{fix}_{X} E \stackrel{\alpha[p]}{\longrightarrow} E^{\prime} .
\end{aligned}
$$

Similar to the assumption on parameterized bisimulation, the assumption that an environment and its behaviors can be described as a deterministic PCCS process is reasonable. Env is the set of environments, Act is the set of actions (identical to the set of actions used in the transition system of processes), 

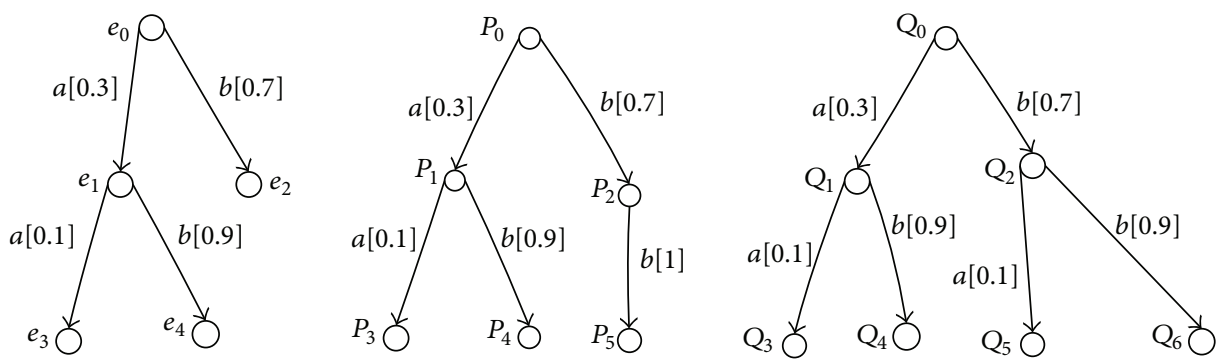

FIGURE 5: $P_{0}$ and $Q_{0}$ are probabilistic bisimulation on the environment $e_{0}$.

and $\Rightarrow$ is a subset of Env $\times$ Act $\times[0,1] \times$ Env, and $e \stackrel{\alpha[p]}{\Rightarrow} e^{\prime}$ means that $e$ may consume the action $\alpha$ with the probability $p$ and after that becomes the environment $e^{\prime}$. fined.

Then, parameterized probabilistic bisimulation is de-

Definition 24. Let $\varepsilon=($ Env, Act, $\Rightarrow)$ be a probabilistic environment transition system. Then an $\varepsilon$-parameterized probabilistic bisimulation, $R$, is an Env-indexed family of binary relations $R_{e} \subseteq \mathrm{DPr} \times \mathrm{DPr}$ for $e \in \mathrm{Env}$, such that whenever $(P, Q) \in R_{e}$ and $e \stackrel{\alpha[p]}{\Rightarrow} f$, then we have the following:

(i) If $P \stackrel{\alpha[p]}{\longrightarrow} P^{\prime}$, then there exists $Q^{\prime} \in \operatorname{DPr}$ s.t. $Q \stackrel{\alpha[p]}{\longrightarrow} Q^{\prime}$ and $\left(P^{\prime}, Q^{\prime}\right) \in R_{f}$.

(ii) If $Q \stackrel{\alpha[p]}{\longrightarrow} Q^{\prime}$, then there exists $P^{\prime} \in$ DPr s.t. $P \stackrel{\alpha[p]}{\longrightarrow} P^{\prime}$ and $\left(P^{\prime}, Q^{\prime}\right) \in R_{f}$.

Define

$$
\sim_{e}^{\mathrm{pr}}=\left\{R_{e} \mid R_{e} \text { is parameterized probabilistic bisimulation over the environment } e\right\} .
$$

Two processes, $P$ and $Q$, are said to be probabilistic bisimulation equivalence on the environment $e \in$ Env if and only if there exists $\mathcal{E}$-parameterized probabilistic bisimulation $R$ such that $(P, Q) \in R_{e}$. We write parameterized probabilistic bisimulation by using the following signal, $P \sim \sim_{e}^{p r} Q$, to distinguish the difference between parameterized bisimulation and parameterized probabilistic bisimulation.

Example 25. Let $\varepsilon, P$, and $Q$ be given in Figure 5. The Envindexed family $R$ is shown as follows:

$$
\begin{aligned}
& R_{e_{0}}=\left\{\left(P_{0}, Q_{0}\right)\right\} \\
& R_{e_{1}}=\left\{\left(P_{1}, Q_{1}\right),\left(P_{2}, Q_{2}\right),\left(P_{1}, Q_{2}\right)\right\} \\
& R_{e_{2}}=\left\{\left(P_{2}, Q_{2}\right)\right\} \\
& R_{e_{3}}=\left\{\left(P_{3}, Q_{3}\right),\left(P_{3}, Q_{5}\right)\right\} \\
& R_{e_{4}}=\left\{\left(P_{4}, Q_{4}\right),\left(P_{4}, Q_{6}\right)\right\} .
\end{aligned}
$$

Then, according to Definition $24, R$ is $\varepsilon$-parameterized probabilistic bisimulation. So, $P_{0} \sim{ }_{e_{0}}^{\mathrm{pr}} Q_{0}, P_{1} \sim{ }_{e_{1}}^{\mathrm{pr}} Q_{1}, P_{1} \sim{ }_{e_{1}}^{\mathrm{pr}} Q_{2}$, but $P_{2}{ }_{e_{1}}^{\mathrm{pr}} Q_{2}, P_{2}{ }_{e_{2}}^{\mathrm{pr}} Q_{2}$.

Next, we try to relax the $\varepsilon$-parameterized probabilistic bisimulation to establish the approximate parameterized probabilistic bisimulation. In [18], there is a rather rich state-space structure and a metric between two processes employs the probabilistic conditional kernels underlying the two stochastic processes. Another metric is based on the dynamical properties of the two processes. Compared with the metric in [18], our model only focuses on the difference of probabilities where two processes can execute the same action.

Definition 26. Let $\varepsilon=($ Env, Act, $\Rightarrow)$ be a probabilistic environment transition system. Consider $\lambda \in[0,1]$. $R$ is an Envindexed family of binary relations, $R_{e} \subseteq \mathrm{DPr} \times \mathrm{DPr}$. If, for any $e \in \operatorname{Env}, e \stackrel{\alpha[r]}{\Rightarrow} e^{\prime}$ and $(P, Q) \in R_{e}$, the following conditions are satisfied:

(1) If $P \stackrel{\alpha[p]}{\longrightarrow} P^{\prime}$, then there exists $Q^{\prime} \in \mathrm{DPr}$ such that $Q \stackrel{\alpha[q]}{\longrightarrow} Q^{\prime}, \max \{|p-r|,|q-r|,|p-q|\} \leq \lambda$, and $\left(P^{\prime}, Q^{\prime}\right) \in R_{e^{\prime}}$.

(2) If $Q \stackrel{\alpha[q]}{\longrightarrow} Q^{\prime}$, then there exists $P^{\prime} \in \mathrm{DPr}$ such that $P \stackrel{\alpha[p]}{\longrightarrow} P^{\prime}, \max \{|p-r|,|q-r|,|p-q|\} \leq \lambda$, and $\left(P^{\prime}, Q^{\prime}\right) \in R_{e^{\prime}}$.

Then we call $R_{e} \lambda$-probabilistic bisimulation. $P$ and $Q$ are said to be $\lambda$-probabilistic bisimilar on the environment $e$ if $\lambda$-probabilistic bisimulation $R_{e}$ exists such that $(P, Q) \in R_{e}$.

Proposition 27. Let $\varepsilon=(E n v$, Act,$\Rightarrow)$ be a probabilistic environment transition system. Consider $\lambda \in[0,1] . R$ is an Env-indexed family of binary relations; $R_{e} \subseteq D \operatorname{Pr} \times D \operatorname{Pr}$ :

(1) If $R$ is a parameterized probabilistic bisimulation, then, for e $\in \mathrm{Env}, R_{e}$ is 0-probabilistic bisimulation. 

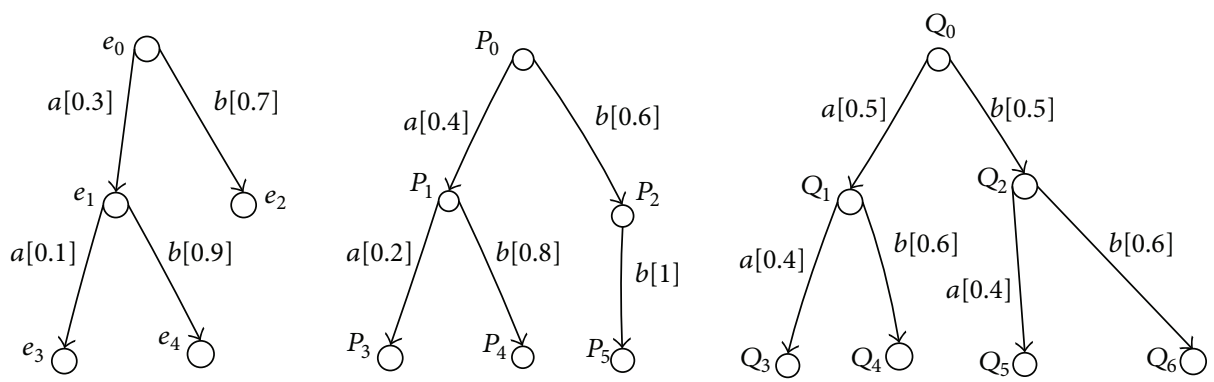

Figure 6: $P_{0}$ and $Q_{0}$ are 0.3-probabilistic bisimulation on the environment $e_{0}$.

(2) For $e \in E n v, R_{e}$ is $\lambda$-probabilistic bisimulation if and only if so is $R_{e}^{-1}$.

(3) For $e \in E n v, R_{e}^{i}$ is a $\lambda_{i}$-probabilistic bisimulation ( $i=$ $1,2)$; then $R_{e}^{1} \circ R_{e}^{2}$ is a $\max \left\{\lambda_{1}, \lambda_{2}\right\}$-probabilistic bisimulation.

(4) For $e \in E n v, R_{e}^{i}$ ( $i \in I$, where $I$ is indexed set) is $\lambda$ probabilistic bisimulation and so is $\bigcup_{i \in I} R_{e}^{i}$.

Definition 28. Let $\varepsilon=(\mathrm{Env}, \mathrm{Act}, \Rightarrow)$ be a probabilistic environment transition system. Consider $\lambda \in[0,1] . R$ is an Envindexed family of binary relations, $R_{e} \subseteq \mathrm{DPr} \times \mathrm{DPr}$; we define

$\sim_{e}^{\mathrm{pr}, \lambda}=\bigcup\left\{R_{e} \mid R_{e}\right.$ is $\lambda$-probabilistic bisimulation $\}$.

If $P$ and $Q$ are said to be $\lambda$-probabilistic bisimilar on the environment $e$, then $\lambda$-probabilistic bisimulation $R_{e}$ exists such that $(P, Q) \in R_{e}$. Thus, we can write $P \sim_{e}^{p r, \lambda} Q$.

Proposition 29. Consider

(1) $\sim_{e}^{p r, \lambda}$ forms a sequence of successively larger relations; that is, $\lambda \leq \lambda^{\prime}$ implies $\sim_{e}^{p r, \lambda} \subset \sim_{e}^{p r, \lambda^{\prime}}$.

(2) $\sim_{e}^{p r, 0}=\bigcap_{\lambda>0} \sim_{e}^{p r, \lambda} ; \sim_{e}^{p r, 0}$ coincides with the parameterized probabilistic bisimulation.

Example 30. Let $\varepsilon, P$, and $Q$ be given in Figure 6. The Envindexed family $R$ is shown as follows:

$$
\begin{aligned}
& R_{e_{0}}=\left\{\left(P_{0}, Q_{0}\right)\right\}, \\
& R_{e_{1}}=\left\{\left(P_{1}, Q_{1}\right),\left(P_{2}, Q_{2}\right),\left(P_{1}, Q_{2}\right)\right\}, \\
& R_{e_{2}}=\left\{\left(P_{2}, Q_{2}\right)\right\}, \\
& R_{e_{3}}=\left\{\left(P_{3}, Q_{3}\right),\left(P_{3}, Q_{5}\right)\right\}, \\
& R_{e_{4}}=\left\{\left(P_{4}, Q_{4}\right),\left(P_{4}, Q_{6}\right)\right\} .
\end{aligned}
$$

Then we can obtain that $P_{0} \sim \sim_{e_{0}}^{\mathrm{pr}, 0.3} Q_{0}, \quad P_{1} \sim \sim_{e_{1}}^{\mathrm{pr}, 0.3} Q_{1}$, $P_{1} \sim{ }_{e_{1}}^{\mathrm{pr}, 0.3} Q_{2}, P_{2} \sim e_{e_{2}}^{\mathrm{pr}, 0.3} Q_{2}$, but $P_{2} \nsim_{e_{1}}^{\mathrm{pr}, 0.3} Q_{2}$.

Theorem 31. Let $\varepsilon=(E n v$, Act, $\Rightarrow)$ be a probabilistic environment transition system; $\lambda \in[0,1]$ :

(1) If $P \sim_{e}^{p r, \lambda} Q$, then $\alpha \cdot P \sim_{\alpha \cdot e}^{p r, \lambda} \alpha \cdot Q$.
(2) If $f$ is isomorphism mapping on Act and $P \sim_{e}^{p r, \lambda} Q$, then $P[f] \sim \underset{f(e)}{p r, \lambda} Q[f]$.

(3) If $P \sim_{e}^{p r, \lambda} Q$ and $R \sim_{f}^{p r, \lambda} T$, then $p P+(1-$ $p) R \sim \underset{p e+(1-p) f}{p r, \lambda} p Q+(1-p) T$.

Proof. (1) It only needs to show there is Env-parameterized probabilistic bisimulation $R ; R_{\alpha \cdot e}$ is $\lambda$-probabilistic bisimulation such that $(\alpha \cdot P, \alpha \cdot Q) \in R_{\alpha \cdot e}$. Since $P \sim_{e}^{p r, \lambda} Q$, Env-parameterized probabilistic bisimulation $S$ exists such that $(P, Q) \in S_{e}$. Then, we construct Env-parameterized probabilistic bisimulation $R$ which is defined as that $R_{\alpha \cdot e}=$ $\left\{(\alpha \cdot P, \alpha \cdot Q) \mid(P, Q) \in S_{e}\right\}$, for any other $e \in$ Env, $R_{e}=S_{e}$. Thus, we need to prove that $R_{\alpha \cdot e}$ is $\lambda$-probabilistic bisimulation. In fact, according to Definition 23, we have that $\alpha \cdot e \stackrel{\alpha[1]}{\Rightarrow} e, \alpha \cdot P \stackrel{\alpha[1]}{\Rightarrow} P$, and $\alpha \cdot Q \stackrel{\alpha[1]}{\Rightarrow} Q$. And $(P, Q) \in S_{e}=R_{e}$, $\max \{|1-1|\}=0 \leq \lambda$. So, $R_{\alpha \cdot e}$ is a $\lambda$-probabilistic bisimulation.

(2) Env-parameterized probabilistic bisimulation $R$ must be presented, and $R_{f(e)}$ is $\lambda$-probabilistic bisimulation such that $(P[f], Q[f]) \in R_{f(e)}$. In fact, since $P \sim_{e}^{p r, \lambda} Q$, Envparameterized probabilistic bisimulation $S$ exists and $S_{e}$ is $\lambda$-probabilistic bisimulation such that $(P, Q) \in S_{e}$. Then, we establish Env-parameterized probabilistic bisimulation $R$, defined as $R_{f(e)}=\left\{(P[f], Q[f]) \mid(P, Q) \in S_{e}\right\}, R_{e}=S_{e}$. Then, $R_{f(e)}$ must be proved to be $\lambda$-probabilistic bisimulation.

In fact, if $f(e) \stackrel{\alpha[r]}{\Rightarrow} f\left(e^{\prime}\right)$, then $e \stackrel{\beta[r]}{\Rightarrow} e^{\prime}$ and $\alpha=f(\beta)$. If $P[f] \stackrel{\alpha[p]}{\longrightarrow} P^{\prime}$, then $P \stackrel{\beta[p]}{\longrightarrow} P^{\prime \prime}$ and $P^{\prime}=P^{\prime \prime}[f], \alpha=f(\beta)$. Since $(P, Q) \in S_{e}, Q^{\prime \prime} \in \operatorname{DPr}$ exists such that $Q \stackrel{\beta[q]}{\longrightarrow} Q^{\prime \prime}$, and $\left(P^{\prime \prime}, Q^{\prime \prime}\right) \in S_{e^{\prime}}$ and $\max \{|r-p|,|r-q|,|p-q|\} \leq \lambda$. Furthermore, according to Definition 23, we can obtain that $Q[f] \stackrel{\alpha[q]}{\longrightarrow} Q^{\prime \prime}[f]=Q^{\prime}$, and $\left(P^{\prime \prime}[f], Q^{\prime \prime}[f]\right) \in R_{f\left(e^{\prime}\right)}$. Thus, $R_{f(e)}$ is $\lambda$-probabilistic bisimulation, so $P[f] \sim_{f(e)}^{\mathrm{pr}, \lambda} Q[f]$.

(3) We need to determine Env-parameterized probabilistic bisimulation $R ; R_{p e+(1-p) f}$ is $\lambda$-probabilistic bisimulation such that $(p P+(1-p) R, p Q+(1-p) T) \in R_{p e+(1-p) f}$. In fact, since $P \sim_{e}^{\mathrm{pr}, \lambda} Q, R \sim_{f}^{\mathrm{pr}, \lambda} T$, so there are Env-parameterized $S^{1}$ and $S^{2}$ such that $(P, Q) \in S_{e}^{1},(R, T) \in S_{f}^{2}$. Next, new Env-parameterized probabilistic bisimulation $R$ is defined as $R_{p e+(1-p) f}=\{(p P+(1-p) R, p Q+(1-p) T) \mid(P, Q) \epsilon$ $S_{e}^{1}$ and $\left.(R, T) \in S_{e}^{2}\right\}, R_{e}=S_{e}^{1}$, and $R_{f}=S_{f}^{2}$. 
If $p e+(1-p) f \stackrel{\alpha[r]}{\Rightarrow} e^{\prime}$, then, according to Definition 23, two cases exist.

Case $1\left(e \stackrel{\alpha[m]}{\Rightarrow} e^{\prime}\right.$ and $\left.r=p m\right)$. If $p P+(1-p) R \stackrel{\alpha[h]}{\longrightarrow} P^{\prime}$, then $P \stackrel{\alpha[l]}{\longrightarrow} P^{\prime}$ or $R \stackrel{\alpha[v]}{\longrightarrow} P^{\prime}$ and $h=p l$ or $h=(1-p) v$. When $P \stackrel{\alpha[l]}{\longrightarrow} P^{\prime}$, since $(P, Q) \in S_{e}^{1}$, so there is $Q \in \mathrm{DPr}$ such that $Q \stackrel{\alpha[k]}{\longrightarrow} Q^{\prime}$ and $\max \{|l-m|,|k-m|,|k-l|\} \leq \lambda$, and $\left(P^{\prime}, Q^{\prime}\right) \in S_{e^{\prime}}^{1}=R_{e^{\prime}}$. So, according to Definition 23, we can get $p Q+(1-p) R \stackrel{\alpha[p k]}{\longrightarrow} Q^{\prime}$ and $\max \{|h-r|,|p k-r|, \mid h-$ $p k \mid\}=p \max \{|l-m|,|k-m|,|l-k|\} \leq p \lambda \leq \lambda$ and $\left(P^{\prime}, Q^{\prime}\right) \in$ $S_{e^{\prime}}^{1}=R_{e^{\prime}}$. When $R \stackrel{\alpha[v]}{\longrightarrow} P^{\prime}$, then since $(R, T) \in S_{e}^{2}$, there exists $T^{\prime} \in \mathrm{DPr}$ such that $T \stackrel{\alpha[u]}{\longrightarrow} T^{\prime}$ and $\left(P^{\prime}, T^{\prime}\right) \in S_{f^{\prime}}^{2}$ and $\max \{|v-m|,|v-u|,|u-m|\} \leq \lambda$. According to Definition 23, we can obtain that $p Q+(1-p) T \stackrel{\alpha[(1-p) u]}{\longrightarrow} T^{\prime}$, so $\max \{\mid(1-$ p) $u-p m|| h-,p m||,(1-p) u-h \mid\}=\max \{|(1-p) u-p m|$, $|(1-p) v-p m|,|(1-p) u-(1-p) v|\} \leq \lambda$, and $\left(P^{\prime}, T^{\prime}\right) \in S_{f^{\prime}}^{2}=$ $R_{f^{\prime}}$.

Case 2 (there is $f \stackrel{\alpha[m]}{\Rightarrow} f^{\prime}$ and $r=(1-p) m$ ). It is similar to Case 1.

Notice that we do not show the proof of the substitutivity law of the synchrony operator $x$ and restrict operator $\lceil$. The main reason is that we can not find a suitable $\lambda$ - $\varepsilon$-parameterized probabilistic bisimulation according to Definition 24. Therefore, our model only uses prefix operator $\alpha$, relabeled operator $[f]$, and sum operator $\sum$ to help us verify the approximate correctness. In the future, I will attempt to find a suitable model to support the substitutivity law.

\section{Conclusion}

In this study, we formalized the approximate correctness of software products. We focused on the approximate version of parameterized bisimulation and extended parameterized bisimulation to probabilistic settings. We presented the definitions of $\lambda$-parameterized bisimulation and $\lambda$ parameterized probabilistic bisimulation over the environment $e$. These approximate versions provide theoretical foundations to verify the degree to which the software is approximately correct. In the future, we will try to find some effective algorithms to realize the verification. The substitutivity laws of $\lambda$-parameterized bisimulation over the environment $e$ and $\lambda$-parameterized probabilistic bisimulation over the environment $e$ are presented.

The modal logical characterizations of $\lambda$-parameterized bisimulation and $\lambda$-parameterized probabilistic bisimulation over the environment $e$ are useful to verify whether or not two processes are approximate under parameterized bisimulation. In the future, we will attempt to establish the modal logical descriptions of $\lambda$-parameterized bisimulation and $\lambda$-parameterized probabilistic bisimulation.

\section{Conflict of Interests}

The author declares that there is no conflict of interests regarding the publication of this paper.

\section{Acknowledgments}

The work is supported by the NSFC (61300048, 61021004), the Anhui Provincial Natural Science Foundation (1308085QF117, 1508085MA14), the Key Natural Science Foundation of Universities of Anhui Province (KJ2014A223, KJ2011A248), the Excellent Young Talents in Universities of Anhui Province, and the Major Teaching Reform Project of Anhui Higher Education Revitalization Plan (2014ZDJY058).

\section{References}

[1] C. X. Shen, H. G. Zhang, and H. M. Wang, "Research and development of trusted computing," Science China: Information Sciences, vol. 40, no. 2, pp. 139-166, 2010.

[2] H. W. Tao and Y. X. Chen, "Another metric model for trustworthiness of softwares based on partition," in Quantitative Logic and Soft Computing 2010, vol. 82 of Advances in Intelligent and Soft Computing, pp. 695-705, Springer, Berlin, Germany, 2010.

[3] K. Liu, Z. G. Shan, J. Wang et al., "Overview on major research plan of trustworthy software," Bulletin of National Natural Science Foundation of China, vol. 3, pp. 145-151, 2008.

[4] K. G. Larsen, U. Fahrenberg, and C. Thrane, "Metrics for weighted transition systems: axiomatization and complexity," Theoretical Computer Science, vol. 412, no. 28, pp. 3358-3369, 2011.

[5] H. Y. Pan, Y. Z. Cao, M. Zhang, and Y. Chen, "Simulation for lattice-valued doubly labeled transition systems," International Journal of Approximate Reasoning, vol. 55, no. 3, pp. 797-811, 2014.

[6] H. Y. Pan, M. Zhang, H. Y. Wu, and Y. Chen, "Quantitative analysis of lattice-valued Kripke structures," Fundamenta Informaticae, vol. 135, no. 3, pp. 269-293, 2014.

[7] M. S. Ying, "Bisimulation indexes and their applications," Theoretical Computer Science, vol. 275, no. 1-2, pp. 1-68, 2002.

[8] M. S. Ying and M. Wirsing, "Approximate bisimilarity," in Algebraic Methodology and Software Technology: 8th International Conference, AMAST 2000 Iowa City, Iowa, USA, May 20-27, 2000 Proceedings, vol. 1816 of Lecture Notes in Computer Science, pp. 309-322, Springer, Berlin, Germany, 2000.

[9] A. Girard and G. J. Pappas, "Approximation metrics for discrete and continuous systems," IEEE Transactions on Automatic Control, vol. 52, no. 5, pp. 782-798, 2007.

[10] T. A. Henzinger, "Quantitative reactive modeling and verification," Computer Science - Research and Development, vol. 28, no. 4, pp. 331-344, 2013.

[11] U. Fahrenberg and A. Legay, "The quantitative linear-timebranching-time spectrum," Theoretical Computer Science, vol. 538, pp. 54-69, 2014.

[12] Y. X. Deng, R. van Glabbeek, M. Hennessy, and C. Morgan, “Testing finitary probabilistic processes," in CONCUR 2009Concurrency Theory, vol. 5710 of Lecture Notes in Computer Science, pp. 274-288, Springer, Berlin, Germany, 2009.

[13] A. Giacalone, C. C. Jou, and S. A. Smolka, "Algebraic reasoning for probabilistic concurrent systems," in Proceedings of the 
Working Conference on Programming Concepts and Methods, IFIP TC2, pp. 443-458, Sea of Gallilee, Israel, 1990.

[14] A. Giacalone, C. C. Jou, and S. A. Smolka, "Probabilistic in processes: a algebraic/operational framework," Tech. Rep. $88 / 20$, Department of Computer Science, SUNY at Stony Brook, New York, NY, USA, 1988.

[15] L. Song, Y. X. Deng, and X. J. Cai, "Towards automatic measurement of probabilistic processes," in Proceedings of the 7th International Conference on Quality Software (QSIC '07), pp. 50-59, October 2007.

[16] Y. X. Deng, T. Chothia, C. Palamidessi, and J. Pang, "Metrics for action-labelled quantitative transition systems," Electronic Notes in Theoretical Computer Science, vol. 153, no. 2, pp. 79-96, 2006.

[17] A. K. Alves de Medeiros, W. M. P. van der Aalst, and A. J. M. M. Weijters, "Quantifying process equivalence based on observed behavior," Data and Knowledge Engineering, vol. 64, no. 1, pp. 55-74, 2008.

[18] A. Abate, "Approximation metrics based on probabilistic bisimulations for general state-space Markov processes: a survey," Electronic Notes in Theoretical Computer Science, vol. 297, pp. 3-25, 2013.

[19] K. G. Larsen and A. Skou, "Bisimulation through probabilistic testing," Information and Computation, vol. 94, no. 1, pp. 1-28, 1991.

[20] J. F. He and T. Hoare, "Equating bisimulation with refinement," Tech. Rep., UNU-IIST, Macau, China, 2003.

[21] Y. F. Ma, M. Zhang, Y. X. Chen, and L. Chen, "Two-thirds simulation indexes and modal logic characterization," Frontiers of Computer Science in China, vol. 5, no. 4, pp. 454-471, 2011.

[22] R. Milner, Communicating and Mobile Systems: The $\pi$-Calculus, Cambridge University Press, Cambridge, UK, 2001.

[23] M. D. Ryan and B. Smyth, "Applied pi-calculus," in Formal Models and Techniques for Analyzing Security Protocols, V. Cortier and S. Kremer, Eds., Ios Press, 2011.

[24] K. G. Larsen, Context-dependent bisimulation between process [Doctor of Philosophy University of Edinburgh], Aalborg University Centre, Strandvejen, Denmark, 1986.

[25] Y. F. Ma and M. Zhang, "Topological construction of parameterized bisimulation limit," Electronic Notes in Theoretical Computer Science, vol. 257, pp. 55-70, 2009.

[26] Y. Ma, M. Zhang, and Y. Chen, "Formal description of software dynamic correctness," Journal of Computer Research and Development, vol. 50, no. 3, pp. 626-635, 2013.

[27] M. Ying, Topology in Processes Calculus: Approximate Correctness and Infinite Evolution of Concurrent Programs, Springer, New York, NY, USA, 2001.

[28] R. Milner, Communication and Concurrency, Prentice Hall, New York, NY, USA, 1989.

[29] G. D. Plotkin, "A structural approach to operational semantics," Tech. Rep. DAIMI FN-19, Computer Science Department, Aarhus University, Aarhus, Denmark, 1981.

[30] R. Engelking, General Topology, Polish Scientific, Warszawa, Poland, 1977.

[31] R. J. van Glabbeek, S. A. Smolka, and B. Steffen, "Reactive, generative, and stratified models of probabilistic processes," Information and Computation, vol. 121, no. 1, pp. 59-80, 1995.

[32] S. A. Smolka and B. Steffen, "Priority as extremal probability," in Proceedings of the Theories of Concurrency: Unification and Extension (CONCUR '90), pp. 456-466, August 1990.

[33] R. Milner, "Calculi for synchrony and asynchrony," Theoretical Computer Science, vol. 25, no. 3, pp. 267-310, 1983.
[34] M. S. Ying, "Additive models of probabilistic processes," Theoretical Computer Science, vol. 275, no. 1-2, pp. 481-519, 2002. 


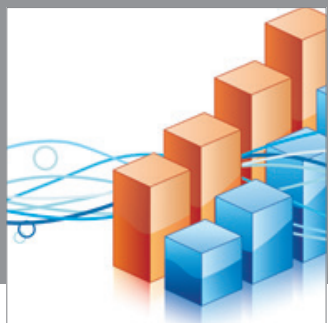

Advances in

Operations Research

mansans

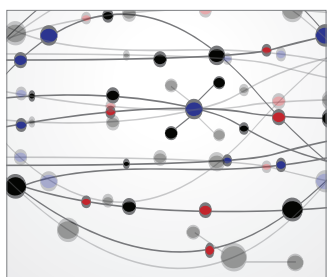

The Scientific World Journal
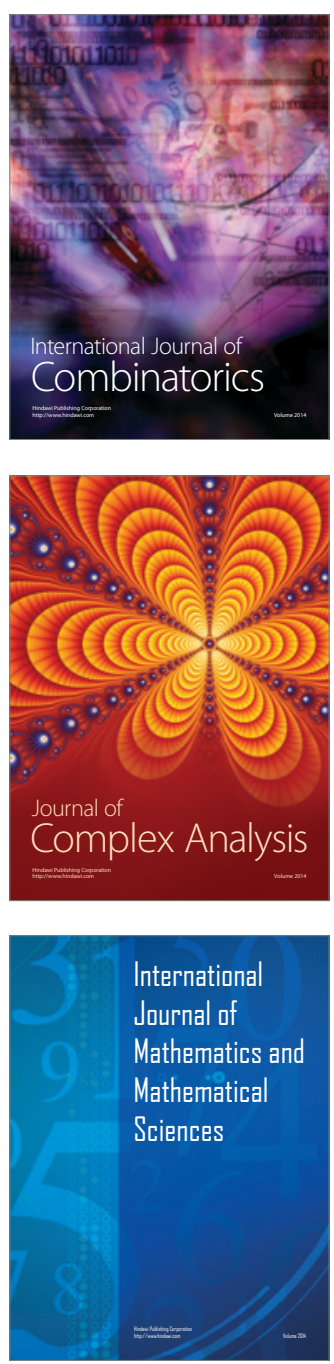
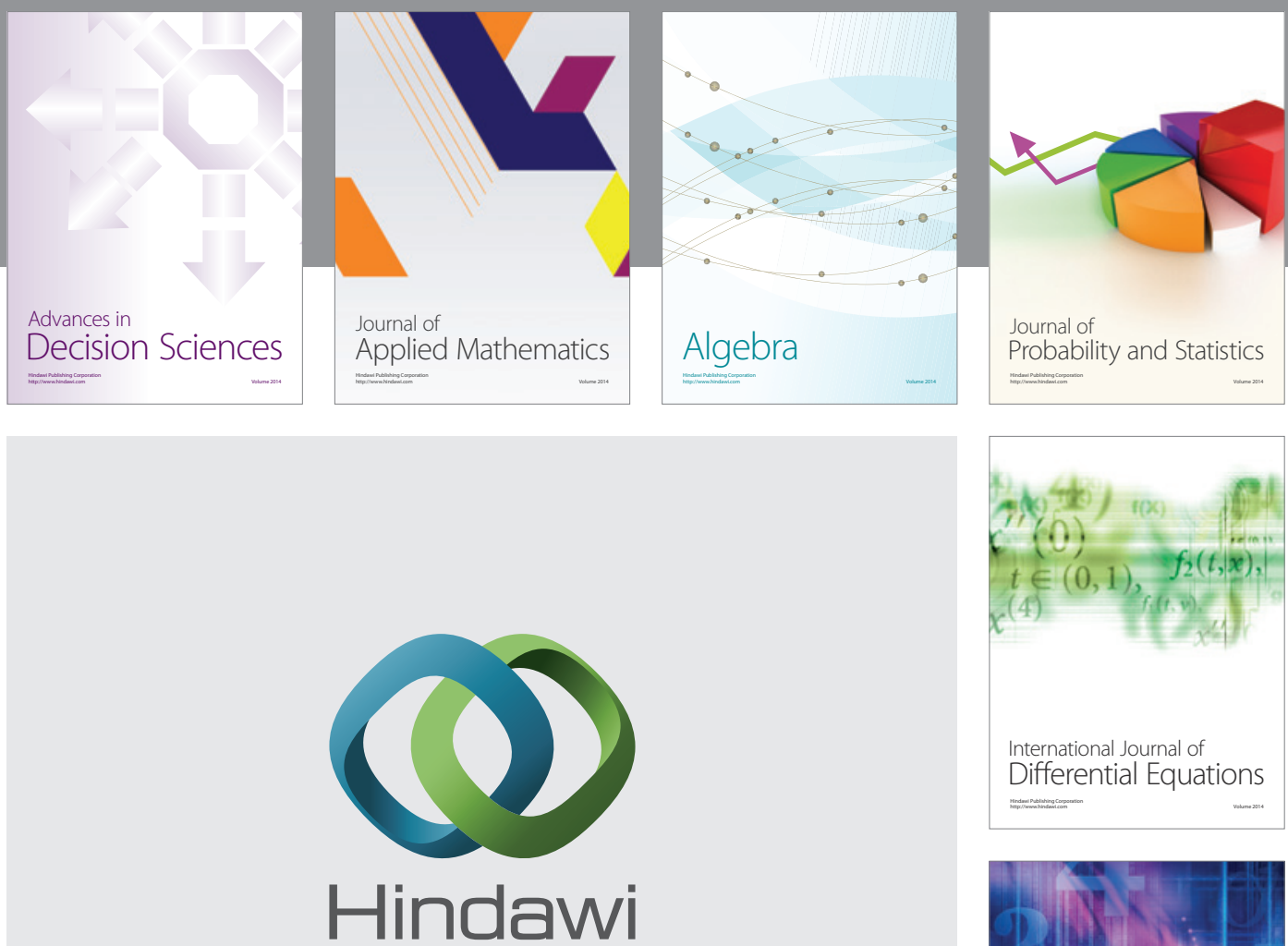

Submit your manuscripts at http://www.hindawi.com
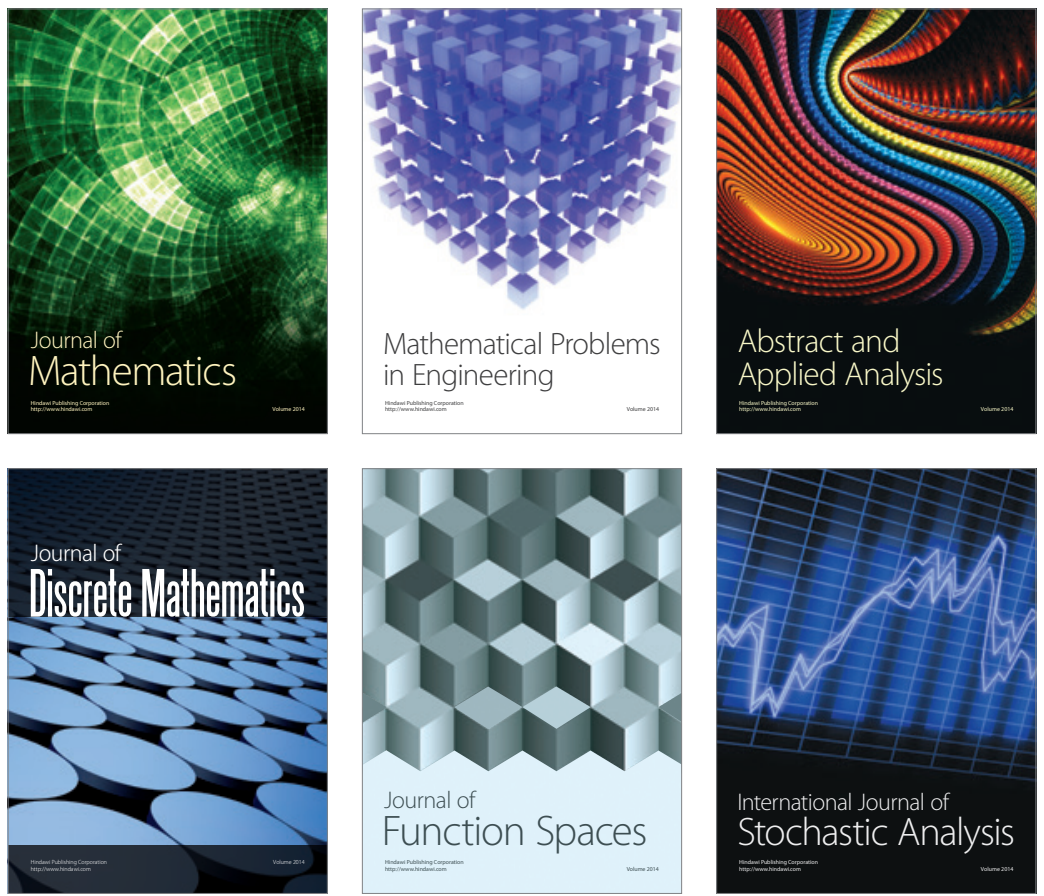

Journal of

Function Spaces

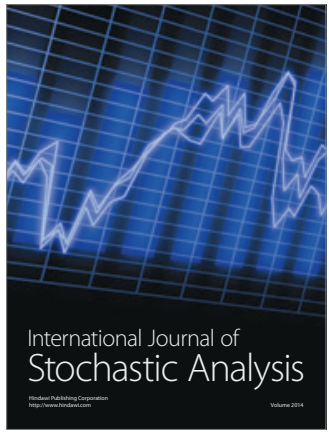

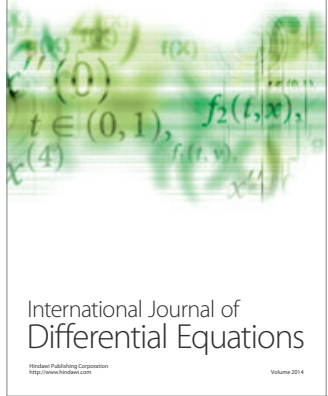
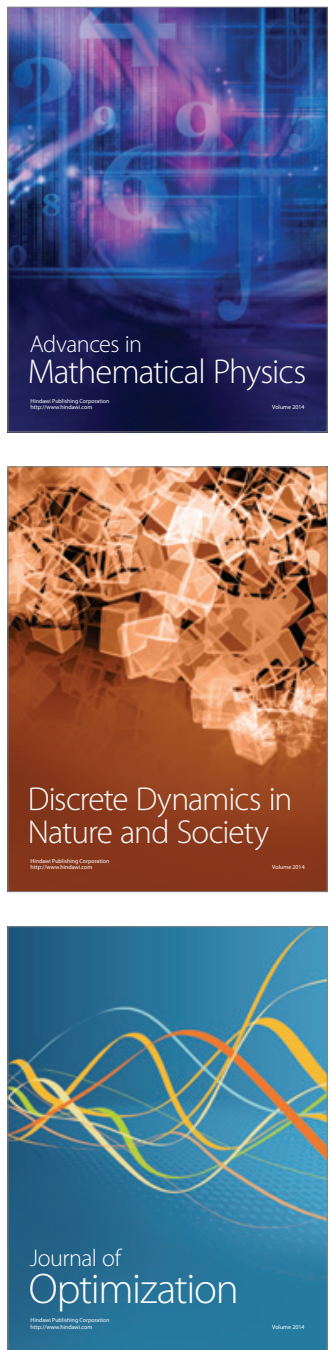